\title{
CONNECTING DISCRETE AND CONTINUOUS LOOKBACK OR HINDSIGHT OPTIONS IN EXPONENTIAL LÉVY MODELS
}

\author{
E. H. A. DIA *** AND \\ D. LAMBERTON, ${ }^{*}$ Université Paris-Est
}

\begin{abstract}
Motivated by the pricing of lookback options in exponential Lévy models, we study the difference between the continuous and discrete supremums of Lévy processes. In particular, we extend the results of Broadie, Glasserman and Kou (1999) to jump diffusion models. We also derive bounds for general exponential Lévy models.
\end{abstract}

Keywords: Exponential Lévy model; lookback option; continuity correction

2010 Mathematics Subject Classification: Primary 60G51; 60J75; 65N15

Secondary $91 \mathrm{G} 20$

\section{Introduction}

The payoff of a lookback option typically depends on the maximum or the minimum of the underlying stock price. The maximum can be evaluated in continuous or discrete time depending on the contract. In the Black-Scholes setting, Broadie et al. [4], [5] derived a number of results relating discrete and continuous path-dependent options (see also [10] for results on diffusion processes). In particular, they obtained continuity correction formulae for lookback, barrier, and hindsight options. The purpose of this paper is to establish similar results for exponential Lévy models. We will focus on lookback or hindsight options, leaving the treatment of barrier options to another paper (see [8] and also [9]).

Our results are based on the analysis of the difference between the discrete and continuous maximums of a Lévy process. In the case of a Lévy process with finite activity and a nonzero Brownian part, we extend (see Theorem 5) the theorem of Asmussen et al. [2] which is the key to the continuity correction formulae for lookback options in Broadie et al. [5]. This allows us to extend these formulae to jump diffusion models. We also establish estimates for the $L_{1}$-norm of the difference between the continuous and discrete maximums of a general Lévy process. These estimates are based on Spitzer's identity, which relates the expectation of the supremum of sums of independent and identically distributed (i.i.d.) random variables to a weighted sum of the expectations of the positive parts of the partial sums. In the case of Lévy processes with finite activity, we derive an expansion up to order $o(1 / n)$, where $n$ is the number of dates in the discrete supremum; see Theorem 1. In the case of infinite activity, we have precise upper bounds (see Theorem 2). We also derive an expansion in the case of Lévy processes with finite variation (see Theorem 3).

The paper is organized as follows. In Section 2 we recall some basic facts about real Lévy processes. In Section 3 we state Spitzer's identity for Lévy processes and use it to analyse the

Received 12 August 2010; revision received 10 June 2011.

* Postal address: Laboratoire d'Analyse et de Mathématiques Appliquées, Université Paris-Est, UMR CNRS 8050,

5 boulevard Descartes, Champs-sur-Marne, 77454 Marne-la-Vallée, France.

** Email address: dia.eha@gmail.com 
expectation of the difference between the continuous and discrete maximums of a general Lévy process. Section 4 is devoted to the extension of the theorem of Asmussen et al. [2]. The last two sections are devoted to financial applications. In Section 5 we derive continuity corrections for lookback options in jump diffusion models, and in Section 6 we give upper bounds for the case of general exponential Lévy models.

\section{Preliminaries}

A real Lévy process $X$ is characterized by its generating triplet $\left(\gamma, \sigma^{2}, \nu\right)$, where $(\gamma, \sigma) \in$ $\mathbb{R} \times \mathbb{R}^{+}$and $v$ is a Radon measure on $\mathbb{R} \backslash\{0\}$ satisfying

$$
\int_{\mathbb{R}}\left(1 \wedge x^{2}\right) v(\mathrm{~d} x)<\infty .
$$

By the Lévy-Itô decomposition, $X$ can be written in the form

$$
X_{t}=\gamma t+\sigma B_{t}+X_{t}^{l}+\lim _{\varepsilon \downarrow 0} \tilde{X}_{t}^{\varepsilon},
$$

with

$$
\begin{gathered}
X_{t}^{l}=\int_{|x|>1, s \in[0, t]} x J_{X}(\mathrm{~d} x \times \mathrm{d} s) \equiv \sum_{0 \leq s \leq t}^{\left|\Delta X_{s}\right| \geq 1} \Delta X_{s}, \\
\tilde{X}_{t}^{\varepsilon}=\int_{\varepsilon \leq|x| \leq 1, s \in[0, t]} x \widetilde{J}_{X}(\mathrm{~d} x \times \mathrm{d} s) \equiv \sum_{0 \leq s \leq t}^{\varepsilon \leq\left|\Delta X_{s}\right|<1} \Delta X_{s}-t \int_{\varepsilon \leq|x| \leq 1} x v(\mathrm{~d} x) .
\end{gathered}
$$

Here $J$ is a Poisson measure on $\mathbb{R} \times[0, \infty)$ with intensity $v(\mathrm{~d} x) \mathrm{d} t, \widetilde{J}_{X}(\mathrm{~d} x \times \mathrm{d} s)=J_{X}(\mathrm{~d} x \times$ $\mathrm{d} s)-v(\mathrm{~d} x) \mathrm{d} s$, and $B$ is a standard Brownian motion. We also have the Lévy-Khinchine formula for the characteristic function of $X_{t}$. Namely,

$$
\mathrm{E}^{\mathrm{i} u X_{t}}=\mathrm{e}^{t \varphi(u)}, \quad u \in \mathbb{R},
$$

where $\varphi$ is given by

$$
\varphi(u)=\mathrm{i} \gamma u-\frac{\sigma^{2} u^{2}}{2}+\int_{\mathbb{R}}\left(\mathrm{e}^{\mathrm{i} u x}-1-\mathrm{i} u x \mathbf{1}_{\{|x| \leq 1\}}\right) v(\mathrm{~d} x) .
$$

We say that $X$ has finite activity if the Lévy measure $v$ is finite $(v(\mathbb{R})<\infty)$. We then have

$$
X_{t}=\gamma_{0} t+\sigma B_{t}+\sum_{i=1}^{N_{t}} Y_{i}
$$

where $N$ is a Poisson process with rate $\lambda=v(\mathbb{R}),\left(Y_{i}\right)_{i \geq 1}$ are i.i.d. random variables with common distribution $v(\mathrm{~d} x) / v(\mathbb{R})$, and

$$
\gamma_{0}=\gamma-\int_{|x| \leq 1} x v(\mathrm{~d} x)
$$

This is a jump diffusion process. If the jump part of $X$ has finite variation (which is equivalent to $\left.\int_{|x| \leq 1}|x| v(\mathrm{~d} x)<\infty\right)$ then

$$
X_{t}=\gamma_{0} t+\sigma B_{t}+\int_{x \in \mathbb{R}, s \in[0, t]} x J_{X}(\mathrm{~d} x \times \mathrm{d} s),
$$

with $\gamma_{0}$ given by (3). Note that $X$ is a finite variation Lévy process if and only if $\sigma=0$ and $\int_{|x| \leq 1}|x| v(\mathrm{~d} x)<\infty$. Moreover, $X$ is integrable if and only if $\int_{|x|>1}|x| v(\mathrm{~d} x)<\infty$. 


\section{Spitzer's identity and applications}

In this section we will first state Spitzer's identity for Lévy processes (we refer the reader to [1, Proposition 4.5, p. 177] for the classical form of Spitzer's identity). Then we will use this result to derive expansions for the error between the continuous and discrete supremums of Lévy processes.

Definition 1. We define

$$
M_{t}^{X}=\sup _{0 \leq s \leq t} X_{s}, \quad M_{t}^{X, n}=\max _{0 \leq k \leq n} X_{k t / n} .
$$

When there is no ambiguity, we can remove the superscript $X$.

Remark 1. Note that $M_{t}$ is integrable for all $t>0$ if and only if $\int_{x>1} x v(\mathrm{~d} x)$ is finite. We also have, for all $\alpha>0, \mathrm{E} \mathrm{e}^{\alpha M_{t}}<\infty$ if and only if $\int_{x>1} \mathrm{e}^{\alpha x} v(\mathrm{~d} x)$ is finite, as can be seen by classical arguments (see [12, Section 25]).

In the setting of Lévy processes, we have the following version of Spitzer's identity.

Proposition 1. If $X$ is a Lévy process with generating triplet $\left(\gamma, \sigma^{2}, v\right)$ satisfying

$$
\int_{x>1} x v(\mathrm{~d} x)<\infty
$$

then

$$
\mathrm{E} M_{t}^{n}=\sum_{k=1}^{n} \frac{\mathrm{E} X_{k t / n}^{+}}{k}, \quad \mathrm{E} M_{t}=\int_{0}^{t} \frac{\mathrm{E} X_{s}^{+}}{s} \mathrm{~d} s .
$$

For the proof of the above result, we need some estimates for E $M_{t}$ with respect to $t$.

Proposition 2. Let $X$ be a Lévy process with generating triplet $\left(\gamma, \sigma^{2}, v\right)$ satisfying

$$
\int_{x>1} x v(\mathrm{~d} x)<\infty
$$

Then

$$
\mathrm{E} M_{t} \leq\left(\gamma^{+}+\int_{x>1} x v(\mathrm{~d} x)\right) t+\left(\sigma \sqrt{\frac{2}{\pi}}+2 \sqrt{\int_{|x| \leq 1} x^{2} v(\mathrm{~d} x)}\right) \sqrt{t}
$$

If, in addition, $\int_{|x| \leq 1}|x| v(\mathrm{~d} x)<\infty$ then

$$
\mathrm{E} M_{t} \leq\left(\gamma_{0}^{+}+\int_{\mathbb{R}^{+}} x v(\mathrm{~d} x)\right) t+\sigma \sqrt{\frac{2}{\pi}} \sqrt{t} .
$$

Proof. We will first prove the second result of the proposition. We have (see (4))

$$
\begin{aligned}
\sup _{0 \leq s \leq t} X_{s} & =\sup _{0 \leq s \leq t}\left(\gamma_{0} s+\sigma B_{s}+\int_{x \in \mathbb{R}, \tau \in[0, s]} x J_{X}(\mathrm{~d} x \times \mathrm{d} \tau)\right) \\
& \leq \gamma_{0}^{+} t+\sigma \sup _{0 \leq s \leq t} B_{s}+\int_{x \in \mathbb{R}^{+}, \tau \in[0, t]} x J_{X}(\mathrm{~d} x \times \mathrm{d} \tau) .
\end{aligned}
$$

So

$$
\mathrm{E} \sup _{0 \leq s \leq t} X_{s} \leq \gamma_{0}^{+} t+\sigma \mathrm{E} \sup _{0 \leq s \leq t} B_{s}+t \int_{\mathbb{R}^{+}} x v(\mathrm{~d} x)
$$


By the reflection theorem we know that $\sup _{0 \leq s \leq t} B_{s}$ has the same distribution as $\left|B_{t}\right|$. Therefore,

$$
\mathrm{E} \sup _{0 \leq s \leq t} B_{s}=\mathrm{E}\left|B_{t}\right|=\sqrt{\frac{2}{\pi}} \sqrt{t}
$$

Hence,

$$
\mathrm{E}\left(\sup _{0 \leq s \leq t} X_{s}\right) \leq\left(\gamma_{0}^{+}+\int_{\mathbb{R}^{+}} x v(\mathrm{~d} x)\right) t+\sigma \sqrt{\frac{2}{\pi}} \sqrt{t} .
$$

Consider now the general case. We define the process $\left(R_{t}\right)_{t \geq 0}$ by

$$
R_{t}=\lim _{\varepsilon \downarrow 0} \tilde{X}_{t}^{\varepsilon}=\lim _{\varepsilon \downarrow 0} \int_{\varepsilon \leq|x| \leq 1, s \in[0, t]} x \widetilde{J}_{X}(\mathrm{~d} x \times \mathrm{d} s) .
$$

We have, using (1),

$$
\mathrm{E}\left(\sup _{0 \leq s \leq t} X_{s}\right) \leq \mathrm{E} \sup _{0 \leq s \leq t}\left(\gamma s+\sigma B_{s}+X_{s}^{l}\right)+\mathrm{E} \sup _{0 \leq s \leq t}\left(R_{s}\right) .
$$

The process $\left(\gamma s+\sigma B_{s}+X_{s}^{l}\right)_{t \geq 0}$ has finite activity and the support of its Lévy measure does not intersect $[-1,1]$, so

$$
\mathrm{E} \sup _{0 \leq s \leq t}\left(\gamma s+\sigma B_{s}+X_{s}^{l}\right) \leq\left(\gamma^{+}+\int_{x>1} x v(\mathrm{~d} x)\right) t+\sigma \sqrt{\frac{2}{\pi}} \sqrt{t} .
$$

Besides, using the Cauchy-Schwarz and Doob inequalities (note that $R$ is a martingale), we obtain

$$
\mathrm{E} \sup _{0 \leq s \leq t}\left(R_{S}\right) \leq 2 \sqrt{t \int_{|x| \leq 1} x^{2} v(\mathrm{~d} x)} .
$$

Hence,

$$
\mathrm{E}\left(\sup _{0 \leq s \leq t} X_{s}\right) \leq\left(\gamma^{+}+\int_{x>1} x v(\mathrm{~d} x)\right) t+\left(\sigma \sqrt{\frac{2}{\pi}}+2 \sqrt{\int_{|x| \leq 1} x^{2} v(\mathrm{~d} x)}\right) \sqrt{t} .
$$

Proof of Proposition 1. By Proposition 2, there exist $c_{1}, c_{2}>0$ such that, for all $t \geq 0$,

$$
\mathrm{E} \sup _{0 \leq s \leq t} X_{s} \leq c_{1} t+c_{2} \sqrt{t}
$$

Thus,

$$
\frac{\mathrm{E} X_{s}^{+}}{s} \leq \frac{\mathrm{E} \sup _{0 \leq \tau \leq s} X_{\tau}}{s} \leq c_{1}+\frac{c_{2}}{\sqrt{s}} .
$$

Since $s \rightarrow 1 / \sqrt{s}$ is integrable on $[0, t]$, so is $s \rightarrow \mathrm{E} X_{s}^{+} / s$. For $s \in(0, t]$, define

$$
f(s)=\frac{\mathrm{E} X_{s}^{+}}{s}, \quad f_{n}(s)=\sum_{k=1}^{n} \mathbf{1}_{((k-1) t / n, k t / n]}(s) f\left(\frac{k t}{n}\right),
$$

so that

$$
\sum_{k=1}^{n} \frac{\mathrm{E} X_{k t / n}^{+}}{k}=\frac{t}{n} \sum_{k=1}^{n} f\left(\frac{k t}{n}\right)=\int_{0}^{t} f_{n}(s) \mathrm{d} s .
$$


We can prove that $f$ is continuous on $(0, t]$. We deduce that $\lim _{n \rightarrow+\infty} f_{n}=f$ almost everywhere (a.e.). We also have, for any $s \in(0, t]$,

$$
\begin{aligned}
\left|f_{n}(s)\right| & \leq \sum_{k=1}^{n} \mathbf{1}_{((k-1) t / n, k t / n]}(s)\left|f\left(\frac{k t}{n}\right)\right| \\
& \leq \sum_{k=1}^{n} \mathbf{1}_{((k-1) t / n, k t / n]}(s)\left(c_{1}+\frac{c_{2}}{\sqrt{k t / n}}\right) \\
& \leq c_{1}+\frac{c_{2}}{\sqrt{s}} .
\end{aligned}
$$

So by dominated convergence we have $\lim _{n \rightarrow+\infty} \sum_{k=1}^{n} \mathrm{E} X_{k t / n}^{+} / k=\int_{0}^{t}\left(\mathrm{E} X_{s}^{+} / s\right) \mathrm{d} s$. On the other hand,

$$
\max _{k=0, \ldots, n} X_{k t / n}=\max \left(0, X_{t / n}, X_{2 t / n}, \ldots, X_{t}\right)=\max \left(X_{t / n}^{+}, X_{2 t / n}^{+}, \ldots, X_{t}^{+}\right) .
$$

Note that, for $k \geq 1$, we have $X_{k t / n}=\sum_{j=1}^{k}\left(X_{j t / n}-X_{(j-1) t / n}\right)$ and the random variables $\left(X_{j t / n}-X_{(j-1) t / n}\right)_{j \geq 1}$ are i.i.d. So by Spitzer's identity we have

$$
\mathrm{E} \max _{k=0, \ldots, n} X_{k t / n}=\sum_{k=1}^{n} \frac{1}{k} \mathrm{E} X_{k t / n}^{+} .
$$

The sequence $\left(\max _{k=0, \ldots, n} X_{k t / n}\right)_{n \geq 0}$ is dominated by $\sup _{0 \leq s \leq t} X_{s}$, so by using the dominated convergence theorem we obtain

$$
\begin{aligned}
\mathrm{E} \sup _{0 \leq s \leq t} X_{s} & =\mathrm{E} \lim _{n \rightarrow+\infty} \max _{k=0, \ldots, n} X_{k t / n} \\
& =\lim _{n \rightarrow+\infty} \mathrm{E} \max _{k=0, \ldots, n} X_{k t / n} \\
& =\lim _{n \rightarrow+\infty} \sum_{k=1}^{n} \frac{1}{k} \mathrm{E} X_{k t / n}^{+} \\
& =\int_{0}^{t} \frac{\mathrm{E} X_{s}^{+}}{s} \mathrm{~d} s .
\end{aligned}
$$

\subsection{Case of finite activity Lévy processes}

The use of Proposition 1 in the finite activity case leads to the following theorem.

Theorem 1. Let $X$ be a finite activity Lévy process satisfying $\int_{x>1} x v(\mathrm{~d} x)<\infty, t>0$, and $n \in \mathbb{N}$.

1. If $\sigma>0$, we have, for $n \rightarrow+\infty$,

$$
\begin{aligned}
\mathrm{E}\left(M_{t}-M_{t}^{n}\right)= & \frac{1}{2 n}\left(\frac{\gamma_{0} t}{2}+\lambda t \mathrm{E} Y_{1}^{+}-\sigma \sqrt{t} \mathrm{E} \phi\left(\frac{\gamma_{0}}{\sigma} \sqrt{t}+\frac{\sum_{i=1}^{N_{t}} Y_{i}}{\sigma \sqrt{t}}\right)\right) \\
& -\frac{1}{2 n} \mathrm{E}\left(\gamma_{0} t+\sum_{i=1}^{N_{t}} Y_{i}\right) \Phi\left(\frac{\gamma_{0}}{\sigma} \sqrt{t}+\frac{\sum_{i=1}^{N_{t}} Y_{i}}{\sigma \sqrt{t}}\right) \\
& -\frac{\sigma \sqrt{t} \zeta(1 / 2)}{\sqrt{2 \pi n}}+o\left(\frac{1}{n}\right) .
\end{aligned}
$$


Here, $\zeta$ is the Riemann zeta function, and $\phi$ and $\Phi$ are the probability density function and the cumulative distribution function of the standard normal distribution.

2. If $\sigma=0$ then $s \rightarrow \mathrm{E} X_{s}^{+} / s$ is absolutely continuous on $[0, t]$ and we have

$$
\mathrm{E}\left(M_{t}-M_{t}^{n}\right)=\frac{1}{2 n}\left(\gamma_{0}^{+} t+\lambda t \mathrm{E} Y_{1}^{+}-\mathrm{E} X_{t}^{+}\right)+o\left(\frac{1}{n}\right)
$$

when $n \rightarrow+\infty$.

Recall that in the case of Brownian motion, Broadie et al. [5] proved (cf. Lemma 3) a result similar to the first point of the above theorem. In the case $\sigma=0$, if $Y_{1}$ has a continuous density function or $\gamma_{0}=0$, the error $o(1 / n)$ is in fact $O\left(1 / n^{2}\right)$ (see [7]). To prove Theorem 1, we need the following more or less elementary lemmas.

Lemma 1. Let $f \in C^{2}[0, t]$. Then

$$
\begin{aligned}
\int_{0}^{t} \frac{1}{\sqrt{x}} f(\sqrt{x}) \mathrm{d} x= & \frac{t}{n} \sum_{k=1}^{n} \frac{1}{\sqrt{k t / n}} f\left(\sqrt{\frac{k t}{n}}\right)-\frac{\sqrt{t} \zeta(1 / 2) f(0)}{\sqrt{n}} \\
& -\frac{\sqrt{t} f(\sqrt{t})-t f^{\prime}(0)}{2 n}+o\left(\frac{1}{n}\right) .
\end{aligned}
$$

Lemma 2. Let $f$ be an absolutely continuous function on $[0, t]$. Then we have

$$
\int_{0}^{t} f(s) \mathrm{d} s-\frac{t}{n} \sum_{k=1}^{n} f\left(\frac{k t}{n}\right)=\frac{t}{2 n}(f(0)-f(t))+o\left(\frac{1}{n}\right) .
$$

The proof of the previous lemma is based on the following result.

Lemma 3. Let $h \in L^{1}([0, t])$. We define the sequence $\left(I_{m}(h)\right)_{m \geq 1}$ by

$$
I_{m}(h)=\sum_{k=1}^{m} \int_{(k-1) t / m}^{k t / m} h(u)\left(u-(k-1) \frac{t}{m}\right) \mathrm{d} u .
$$

Then we have

$$
\lim _{m \rightarrow+\infty} m I_{m}(h)=\frac{t}{2} \int_{0}^{t} h(u) \mathrm{d} u .
$$

Proof. Consider first the case where $h \in C([0, t])$. By the variable substitutions $v=$ $u-(k-1) t / m$, then $w=m v$, we obtain

$$
\begin{aligned}
I_{m}(h) & =\sum_{k=1}^{m} \int_{0}^{t / m} h\left(v+(k-1) \frac{t}{m}\right) v \mathrm{~d} v \\
& =\sum_{k=1}^{m} \int_{0}^{t} h\left(\frac{w}{m}+(k-1) \frac{t}{m}\right) \frac{w}{m} \frac{\mathrm{d} w}{m} \\
& =\frac{1}{m} \int_{0}^{t} \frac{1}{m} \sum_{k=1}^{m} h\left(\frac{w}{m}+(k-1) \frac{t}{m}\right) w \mathrm{~d} w .
\end{aligned}
$$


But $h$ is continuous and, for $w \in[0, t]$, we have $w / m+(k-1) t / m \in[(k-1) t / m, k t / m]$, so

$$
\lim _{m \rightarrow+\infty} \frac{t}{m} \sum_{k=1}^{m} h\left(\frac{w}{m}+(k-1) \frac{t}{m}\right)=\int_{0}^{t} h(s) \mathrm{d} s .
$$

Hence,

$$
\lim _{m \rightarrow+\infty} m I_{m}(h)=\int_{0}^{t}\left(\frac{1}{t} \int_{0}^{t} h(s) \mathrm{d} s\right) w \mathrm{~d} w=\frac{t}{2} \int_{0}^{t} h(s) \mathrm{d} s .
$$

Consider now the case where $h$ is integrable on $[0, t]$. Then there exists a sequence of functions $\left(h_{n}\right)_{n \geq 0}$ in $C([0, t])$ such that

$$
\lim _{n \rightarrow+\infty} \int_{0}^{t}\left|h(u)-h_{n}(u)\right| \mathrm{d} u=0 .
$$

So we have

$$
\begin{aligned}
u_{m}^{n}: & =\left|m I_{m}\left(h_{n}\right)-m \sum_{k=1}^{m} \int_{(k-1) t / m}^{k t / m} h(u)\left(u-(k-1) \frac{t}{m}\right) \mathrm{d} u\right| \\
& =\left|m \sum_{k=1}^{m} \int_{(k-1) t / m}^{k t / m}\left(h_{n}(u)-h(u)\right)\left(u-(k-1) \frac{t}{m}\right) \mathrm{d} u\right| \\
& \leq m \sum_{k=1}^{m} \int_{(k-1) t / m}^{k t / m}\left|h_{n}(u)-h(u)\right|\left|u-(k-1) \frac{t}{m}\right| \mathrm{d} u \\
& \leq t \sum_{k=1}^{m} \int_{(k-1) t / m}^{k t / m}\left|h_{n}(u)-h(u)\right| \mathrm{d} u \\
& \leq t \int_{0}^{t}\left|h_{n}(u)-h(u)\right| \mathrm{d} u .
\end{aligned}
$$

The convergence (with respect to $m$ ) of $m I_{m}\left(h_{n}\right)$ is uniform. Hence, by the limits inversion theorem,

$$
\begin{aligned}
& \lim _{m \rightarrow+\infty} \lim _{n \rightarrow+\infty} m I_{m}\left(h_{n}\right)=\lim _{n \rightarrow+\infty} \lim _{m \rightarrow+\infty} m I_{m}\left(h_{n}\right) \\
& \Longrightarrow \lim _{m \rightarrow+\infty} m I_{m}(h)=\lim _{n \rightarrow+\infty} \frac{t}{2} \int_{0}^{t} h_{n}(u) \mathrm{d} u \\
& \Longrightarrow \quad \lim _{m \rightarrow+\infty} m I_{m}(h)=\frac{t}{2} \int_{0}^{t} h(u) \mathrm{d} u .
\end{aligned}
$$

Proof of Lemma 2. Let $h$ be the a.e. derivative of $f$. We have

$$
\begin{aligned}
\int_{0}^{t} f(s) \mathrm{d} s-\frac{t}{n} \sum_{k=1}^{n} f\left(\frac{k t}{n}\right) & =\sum_{k=1}^{n} \int_{(k-1) t / n}^{k t / n}\left(f(s)-f\left(\frac{k t}{n}\right)\right) \mathrm{d} s \\
& =-\sum_{k=1}^{n} \int_{(k-1) t / n}^{k t / n} \int_{s}^{k t / n} h(u) \mathrm{d} u \mathrm{~d} s \\
& =-\sum_{k=1}^{n} \int_{(k-1) t / n}^{k t / n} \int_{(k-1) t / n}^{u} h(u) \mathrm{d} s \mathrm{~d} u \quad \text { (by Fubini). }
\end{aligned}
$$


Thus,

$$
\begin{aligned}
\int_{0}^{t} f(s) \mathrm{d} s-\frac{t}{n} \sum_{k=1}^{n} f\left(\frac{k t}{n}\right) & =-\sum_{k=1}^{n} \int_{(k-1) t / n}^{k t / n} h(u)\left(u-(k-1) \frac{t}{n}\right) \mathrm{d} u \\
& =-\frac{t}{2 n} \int_{0}^{t} h(u) \mathrm{d} u+o\left(\frac{1}{n}\right) \quad \text { (by Lemma 3) } \\
& =-\frac{t}{2 n}(f(t)-f(0))+o\left(\frac{1}{n}\right) \\
& =\frac{t}{2 n}(f(0)-f(t))+o\left(\frac{1}{n}\right) .
\end{aligned}
$$

Proof of Lemma 1. We consider first the case $t=1$. The case $t \neq 1$ will be deduced by a variable substitution. We have

$$
\frac{1}{\sqrt{x}} f(\sqrt{x})=\frac{f(0)}{\sqrt{x}}+\frac{f(\sqrt{x})-f(0)}{\sqrt{x}} .
$$

Set

$$
g(x)=\frac{f(\sqrt{x})-f(0)}{\sqrt{x}} .
$$

The function $g$ can be extended to a continuous function on $[0,1]$, and $\lim _{x \rightarrow 0} g(x)=f^{\prime}(0)$. Furthermore, $g$ is differentiable on $(0,1]$ and

$$
g^{\prime}(x)=\frac{f(0)-f(\sqrt{x})+\sqrt{x} f^{\prime}(\sqrt{x})}{2 x^{3 / 2}} .
$$

The function $g^{\prime}$ is integrable on $[0,1]$, so $g$ is absolutely continuous. Thus,

$$
\begin{aligned}
\varepsilon_{n}(f) & =\int_{0}^{1} \frac{f(0)}{\sqrt{x}} \mathrm{~d} x+\int_{0}^{1} g(x) \mathrm{d} x-\frac{1}{n} \sum_{k=1}^{n} \frac{f(0)}{\sqrt{k / n}}-\frac{1}{n} \sum_{k=1}^{n} g\left(\frac{k}{n}\right) \\
& =f(0)\left(\int_{0}^{1} \frac{1}{\sqrt{x}} \mathrm{~d} x-\frac{1}{n} \sum_{k=1}^{n} \frac{1}{\sqrt{k / n}}\right)+\left(\int_{0}^{1} g(x) \mathrm{d} x-\frac{1}{n} \sum_{k=1}^{n} g\left(\frac{k}{n}\right)\right)
\end{aligned}
$$

from [11] (see page 538) and using Lemma 2, we obtain

$$
\begin{aligned}
\varepsilon_{n}(f) & =f(0)\left(-\frac{\zeta(1 / 2)}{\sqrt{n}}-\frac{1}{2 n}+O\left(\frac{1}{n^{2}}\right)\right)+\frac{g(0)}{2 n}-\frac{g(1)}{2 n}+o\left(\frac{1}{n}\right) \\
& =-\frac{\zeta(1 / 2)}{\sqrt{n}} f(0)-\frac{f(0)}{2 n}-\frac{f(1)-f^{\prime}(0)-f(0)}{2 n}+o\left(\frac{1}{n}\right) \\
& =-\frac{\zeta(1 / 2) f(0)}{\sqrt{n}}-\frac{f(1)-f^{\prime}(0)}{2 n}+o\left(\frac{1}{n}\right) .
\end{aligned}
$$

Proof of Theorem 1. We know from Proposition 1 that

$$
\mathrm{E}\left(\sup _{0 \leq s \leq t} X_{s}-\max _{k=0, \ldots, n} X_{k t / n}\right)=\int_{0}^{t} \frac{\mathrm{E} X_{s}^{+}}{s} \mathrm{~d} s-\frac{t}{n} \sum_{k=1}^{n} \frac{\mathrm{E} X_{k t / n}^{+}}{k t / n} .
$$


So we need to study the smoothness of the function $s \mapsto \mathrm{E} X_{s}^{+} / s$, and conclude with Lemma 1 and Lemma 2.

Case 1: $\sigma>0$ and $\mathrm{E} Y_{1}^{+}<\infty$. Let $U$ be a normal random variable with mean $\gamma$ and variance $\sigma^{2}$. By an easy computation we obtain

$$
\mathrm{E} U^{+}=\sigma \phi\left(\frac{\gamma}{\sigma}\right)+\gamma \Phi\left(\frac{\gamma}{\sigma}\right)
$$

So, for any $s>0$, we have, by conditioning with respect to the jump part of the process $X$,

$$
\mathrm{E} \frac{X_{s}^{+}}{s}=\mathrm{E} \frac{\sigma}{\sqrt{s}} \phi\left(\frac{\gamma_{0}}{\sigma} \sqrt{s}+\frac{\sum_{i=1}^{N_{s}} Y_{i}}{\sigma \sqrt{s}}\right)+\mathrm{E}\left(\gamma_{0}+\frac{\sum_{i=1}^{N_{s}} Y_{i}}{s}\right) \Phi\left(\frac{\gamma_{0}}{\sigma} \sqrt{s}+\frac{\sum_{i=1}^{N_{s}} Y_{i}}{\sigma \sqrt{s}}\right) .
$$

Let $f$ and $g$ be the functions defined by

$$
f(s)=\mathrm{E} \phi\left(\frac{\gamma_{0}}{\sigma} s+\frac{\sum_{i=1}^{N_{s^{2}}} Y_{i}}{\sigma s}\right), \quad g(s)=\mathrm{E}\left(\frac{\gamma_{0}}{\sigma} s+\frac{\sum_{i=1}^{N_{s^{2}}} Y_{i}}{\sigma s}\right) \Phi\left(\frac{\gamma_{0}}{\sigma} s+\frac{\sum_{i=1}^{N_{s^{2}}} Y_{i}}{\sigma s}\right),
$$

so that

$$
\mathrm{E} \frac{X_{s}^{+}}{s}=\frac{\sigma}{\sqrt{s}} f(\sqrt{s})+\frac{\sigma}{\sqrt{s}} g(\sqrt{s}) .
$$

If $f$ and $g$ can be extended as $C^{2}$ functions on $[0, t]$ then, using Lemma 1 , we obtain the first part of the theorem. By [6, Proposition 9.5] we have

$$
f(s)=\mathrm{E} s^{2 N_{1}} \mathrm{e}^{-\lambda\left(s^{2}-1\right)} \phi\left(\frac{\gamma_{0}}{\sigma} s+\frac{\sum_{i=1}^{N_{1}} Y_{i}}{\sigma s}\right) .
$$

So, the function $f$ has the same regularity as $\tilde{f}$ defined by

$$
\tilde{f}(s)=\mathrm{E} s^{2 N_{1}} \phi\left(\mu s+\frac{\sum_{i=1}^{N_{1}} Y_{i}}{\sigma s}\right),
$$

where $\mu=\gamma_{0} / \sigma$. For $x \in \mathbb{R}$, we define the function

$$
s \mapsto h(s, x)=\phi\left(\mu s+\frac{x}{s}\right) .
$$

We then have

$$
\tilde{f}(s)=\mathrm{E} s^{2 N_{1}} h\left(s, \frac{\sum_{i=1}^{N_{1}} Y_{i}}{\sigma}\right)
$$

Note that

$$
0 \leq h(s, x) \leq \frac{1}{\sqrt{2 \pi}}
$$

and

$$
h(s, x)=\frac{1}{\sqrt{2 \pi}} \exp \left(-\frac{1}{2}\left(\mu s+\frac{x}{s}\right)^{2}\right)=\frac{1}{\sqrt{2 \pi}} \exp \left(-\frac{\mu^{2} s^{2}}{2}\right) \exp \left(-\mu x-\frac{x^{2}}{2 s^{2}}\right) .
$$


Using the inequality $-\mu x \leq \mu^{2} s^{2}+x^{2} / 4 s^{2}$, we obtain

$$
h(s, x) \leq \frac{1}{\sqrt{2 \pi}}\left(\mathrm{e}^{\mu^{2} s^{2} / 2} \mathrm{e}^{-x^{2} / 4 s^{2}} \wedge 1\right) .
$$

Moreover, we have

$$
\frac{\partial}{\partial s} h(s, x)=\left(\frac{x^{2}}{s^{3}}-\mu^{2} s\right) h(s, x)
$$

and

$$
\frac{\partial^{2}}{\partial s^{2}} h(s, x)=\left(-\frac{3 x^{2}}{s^{4}}-\mu^{2}\right) \phi\left(\mu s+\frac{x}{s}\right)+\left(\frac{x^{2}}{s^{3}}-\mu^{2} s\right)^{2} \phi\left(\mu \sqrt{s}+\frac{x}{\sqrt{s}}\right) .
$$

Using (5), we obtain

$$
\left|\frac{\partial}{\partial s} h(s, x)\right| \leq \frac{\mu^{2} s}{\sqrt{2 \pi}}+\frac{x^{2}}{s^{3} \sqrt{2 \pi}} \mathrm{e}^{\mu^{2} s^{2} / 2} \mathrm{e}^{-x^{2} / 4 s^{2}} \leq \frac{\mu^{2} s}{\sqrt{2 \pi}}+\frac{C_{1} \mathbf{1}_{\{x \neq 0\}}}{s} \mathrm{e}^{\mu^{2} s^{2} / 2},
$$

where $C_{1}=\sup _{y>0}\left(y^{2} \mathrm{e}^{-y^{2} / 4} / \sqrt{2 \pi}\right)$. Using (5) again and the fact that $\left(x^{2} / s^{3}-\mu^{2} s\right)^{2} \leq$ $2\left(x^{4} / s^{6}+\mu^{4} s^{2}\right)$, we obtain

$$
\begin{aligned}
\left|\frac{\partial^{2}}{\partial s^{2}} h(s, x)\right| & \leq\left(\mu^{2}+2 \mu^{4} s^{2}\right) h(s, x)+\left(\frac{3 x^{2}}{s^{4}}+2 \frac{x^{4}}{s^{6}}\right) h(s, x) \\
& \leq \frac{\mu^{2}+2 \mu^{4} s^{2}}{\sqrt{2 \pi}}+\frac{C_{2} \mathbf{1}_{\{x \neq 0\}}}{s^{2}} \mathrm{e}^{\mu^{2} s^{2} / 2},
\end{aligned}
$$

where $C_{2}=\sup _{y>0}\left(\left(3 y^{2}+2 y^{4}\right) \mathrm{e}^{-y^{2} / 4} / \sqrt{2 \pi}\right)$. Hence,

$$
\frac{\partial}{\partial s}\left(s^{2 N_{1}} h\left(s, \frac{\sum_{i=1}^{N_{1}} Y_{i}}{\sigma}\right)\right)=2 N_{1} s^{2 N_{1}-1} h\left(s, \frac{\sum_{i=1}^{N_{1}} Y_{i}}{\sigma}\right)+s^{2 N_{1}} \frac{\partial}{\partial s} h\left(s, \frac{\sum_{i=1}^{N_{1}} Y_{i}}{\sigma}\right) .
$$

Thus,

$$
\left|\frac{\partial}{\partial s}\left(s^{2 N_{1}} h\left(s, \frac{\sum_{i=1}^{N_{1}} Y_{i}}{\sigma}\right)\right)\right| \leq \frac{2 N_{1} s^{2 N_{1}-1}}{\sqrt{2 \pi}}+\frac{\mu^{2} s^{2 N_{1}-1}}{\sqrt{2 \pi}}+C_{1} \mathbf{1}_{\left\{N_{1}>0\right\}} s^{2 N_{1}-1} \mathrm{e}^{\mu^{2} s^{2} / 2} .
$$

We deduce that $\tilde{f}$ is continuously differentiable, and

$$
\tilde{f}^{\prime}(s)=\mathrm{E}\left(2 N_{1} s^{2 N_{1}-1} h\left(s, \frac{\sum_{i=1}^{N_{1}} Y_{i}}{\sigma}\right)+s^{2 N_{1}} \frac{\partial}{\partial s} h\left(s, \frac{\sum_{i=1}^{N_{1}} Y_{i}}{\sigma}\right)\right) .
$$

Similarly,

$$
\begin{aligned}
\frac{\partial^{2}}{\partial s^{2}}\left(s^{2 N_{1}} h\left(s, \frac{\sum_{i=1}^{N_{1}} Y_{i}}{\sigma}\right)\right)= & 2 N_{1}\left(2 N_{1}-1\right) s^{2 N_{1}-2} h\left(s, \frac{\sum_{i=1}^{N_{1}} Y_{i}}{\sigma}\right) \\
& +4 N_{1} s^{2 N_{1}-1} \frac{\partial}{\partial s} h\left(s, \frac{\sum_{i=1}^{N_{1}} Y_{i}}{\sigma}\right)+s^{2 N_{1}} \frac{\partial^{2}}{\partial s^{2}} h\left(s, \frac{\sum_{i=1}^{N_{1}} Y_{i}}{\sigma}\right) .
\end{aligned}
$$


But

$$
\begin{aligned}
& \left|2 N_{1}\left(2 N_{1}-1\right) s^{2 N_{1}-2} h\left(s, \frac{\sum_{i=1}^{N_{1}} Y_{i}}{\sigma}\right)\right| \leq \frac{2 N_{1}\left(2 N_{1}-1\right) s^{2 N_{1}-2}}{\sqrt{2 \pi}}, \\
& \left|42 N_{1} s^{2 N_{1}-1} \frac{\partial}{\partial s}\left(s, \frac{\sum_{i=1}^{N_{1}} Y_{i}}{\sigma}\right)\right| \leq \frac{4 N_{1}\left(2 N_{1}-1\right) s^{N_{1}}}{\sqrt{2 \pi}} \\
& +4 N_{1}\left(2 N_{1}-1\right) s^{2 N_{1}-2} C_{1} \mathbf{1}_{\left\{N_{1}>0\right\}} \mathrm{e}^{\mu^{2} s^{2} / 2}, \\
& \left|s^{2 N_{1}} \frac{\partial^{2}}{\partial s^{2}} h\left(s, \frac{\sum_{i=1}^{N_{1}} Y_{i}}{\sigma}\right)\right| \leq \frac{\mu^{2}+2 \mu^{4} s^{2}}{\sqrt{2 \pi}} s^{2 N_{1}}+C_{2} \mathbf{1}_{\left\{N_{1}>0\right\}} s^{2 N_{1}-2} \mathrm{e}^{\mu^{2} s^{2} / 2} \text {. }
\end{aligned}
$$

We deduce that $\tilde{f}$ is twice differentiable on $[0, t]$ and

$$
\begin{aligned}
\tilde{f}^{\prime \prime}(s)= & \mathrm{E}\left(2 N_{1}\left(2 N_{1}-1\right) s^{2 N_{1}-2} h\left(s, \frac{\sum_{i=1}^{N_{1}} Y_{i}}{\sigma}\right)+4 N_{1} s^{2 N_{1}-1} \frac{\partial}{\partial s} h\left(s, \frac{\sum_{i=1}^{N_{1}} Y_{i}}{\sigma}\right)\right) \\
& +\mathrm{E}\left(s^{2 N_{1}} \frac{\partial^{2}}{\partial s^{2}} h\left(s, \frac{\sum_{i=1}^{N_{1}} Y_{i}}{\sigma}\right)\right) .
\end{aligned}
$$

Hence, $f$ is in $C^{2}[0, t]$, and we verify that $f(0)=1 / \sqrt{2 \pi}$ and $f^{\prime}(0)=0$. On the other hand, the function $g$ can be written in the following form (see [6, Proposition 9.5]):

$$
g(s)=\mathrm{E} s^{2 N_{1}} \mathrm{e}^{-\lambda\left(s^{2}-1\right)}\left(\frac{\gamma_{0}}{\sigma} s+\frac{\sum_{i=1}^{N_{1}} Y_{i}}{\sigma s}\right) \Phi\left(\frac{\gamma_{0}}{\sigma} s+\frac{\sum_{i=1}^{N_{1}} Y_{i}}{\sigma s}\right) .
$$

With the same reasoning we can prove that $g$ is in $C^{2}[0, t]$, and satisfies $g(0)=0$ and $g^{\prime}(0)=\lambda \mathrm{E} Y_{1}^{+} / \sigma+\gamma_{0} / 2 \sigma$. This proves the first part of the theorem.

Case 2: $\sigma=0$ and $\mathrm{E} Y_{1}^{+}<\infty$. We have

$$
\frac{\mathrm{E} X_{s}^{+}}{s}=\gamma_{0}^{+} \mathrm{e}^{-\lambda s}+\mathrm{e}^{-\lambda s} \sum_{n=1}^{+\infty} \frac{\lambda^{n} s^{n-1}}{n !} \mathrm{E}\left(\gamma_{0} s+\sum_{i=1}^{n} Y_{i}\right)^{+}
$$

Observe that, for any positive integer $n$, the function $s \mapsto \mathrm{E}\left(\gamma_{0} s+\sum_{i=1}^{n} Y_{i}\right)^{+}$is absolutely continuous. So is $s \mapsto \lambda^{n} s^{n-1} \mathrm{E}\left(\gamma_{0} s+\sum_{i=1}^{n} Y_{i}\right)^{+} / n$ !. If we call $h_{n}$ its a.e. derivative then, for any $n \geq 2$,

$$
h_{n}(s)=\gamma_{0} \frac{\lambda^{n} s^{n-1}}{n !} \mathrm{P}\left[\gamma_{0} s+\sum_{i=1}^{n} Y_{i} \geq 0\right]+\frac{n-1}{n !} \lambda^{n} s^{n-2} \mathrm{E}\left(\gamma_{0} s+\sum_{i=1}^{n} Y_{i}\right)^{+}
$$

so that, for $s \in[0, t]$,

$$
\left|h_{n}(s)\right| \leq\left|\gamma_{0}\right| \frac{\lambda^{n} t^{n-1}}{n !}+\frac{n-1}{n !} \lambda^{n} t^{n-2}\left(\left|\gamma_{0}\right| t+n \mathrm{E} Y_{1}^{+}\right)
$$

Hence, the normal convergence of $\sum h_{n}$ on $[0, t]$, and, thus, the absolute continuity of $\mathrm{E} X_{s}^{+} / s$ 
on $[0, t]$. So, by Proposition 1 and Lemma 2,

$$
\begin{aligned}
\mathrm{E}\left(\sup _{0 \leq s \leq t} X_{s}-\max _{k=0, \ldots, n} X_{k t / n}\right) & =\int_{0}^{t} \frac{\mathrm{E} X_{s}^{+}}{s} \mathrm{~d} s-\frac{t}{n} \sum_{k=1}^{n} \frac{\mathrm{E} X_{k t / n}^{+}}{k t / n} \\
& =\frac{t}{2 n}\left(\lim _{s \rightarrow 0^{+}} \frac{\mathrm{E} X_{s}^{+}}{s}-\frac{\mathrm{E} X_{t}^{+}}{t}\right)+o\left(\frac{1}{n}\right) \\
& =\frac{1}{2 n}\left(\left(\gamma_{0}^{+}+\lambda \mathrm{E} Y_{1}^{+}\right) t-\mathrm{E} X_{t}^{+}\right)+o\left(\frac{1}{n}\right) \\
& =\frac{1}{2 n}\left(\gamma_{0}^{+} t+\lambda t \mathrm{E} Y_{1}^{+}-\mathrm{E} X_{t}^{+}\right)+o\left(\frac{1}{n}\right)
\end{aligned}
$$

\subsection{Case of infinite activity Lévy processes}

In the case of Lévy processes with infinite activity, we cannot use (2). So the method used in Theorem 1 does not work anymore and we must use another approach.

Theorem 2. Let $X$ be an integrable Lévy process with generating triplet $\left(\gamma, \sigma^{2}, v\right)$. Then

1. if $\sigma>0$,

$$
\mathrm{E}\left(M_{t}-M_{t}^{n}\right)=O\left(\frac{1}{\sqrt{n}}\right),
$$

2. if $\sigma=0$,

$$
\mathrm{E}\left(M_{t}-M_{t}^{n}\right)=o\left(\frac{1}{\sqrt{n}}\right),
$$

3. if $\sigma=0$ and $\int_{|x| \leq 1}|x| v(\mathrm{~d} x)<\infty$,

$$
\mathrm{E}\left(M_{t}-M_{t}^{n}\right)=O\left(\frac{\log (n)}{n}\right) .
$$

To prove result 2 of Theorem 2, we will use the lemma below.

Lemma 4. Let $X$ be an integrable Lévy process with generating triplet $(\gamma, 0, v)$. Then we have

$$
\mathrm{E} X_{t}^{+}=o(\sqrt{t})
$$

when $t \rightarrow 0$.

The proof of this lemma is quite standard, and is left to the reader. For more details, see [7].

Proof of Theorem 2. With the notation $\delta=t / n$, we have, using Proposition 1,

$$
\begin{aligned}
\mathrm{E}\left(M_{t}-M_{t}^{n}\right) & =\int_{0}^{t} \frac{\mathrm{E} X_{s}^{+}}{s} \mathrm{~d} s-\sum_{k=1}^{n} \frac{\mathrm{E} X_{k \delta}^{+}}{k} \\
& =\sum_{k=1}^{n} \int_{(k-1) \delta}^{k \delta} \frac{\mathrm{E} X_{s}^{+}}{s} \mathrm{~d} s-\sum_{k=1}^{n} \int_{(k-1) \delta}^{k \delta} \frac{\mathrm{E} X_{k \delta}^{+}}{k \delta} \mathrm{d} s \\
& =\int_{0}^{\delta}\left(\frac{\mathrm{E} X_{s}^{+}}{s}-\frac{\mathrm{E} X_{\delta}^{+}}{\delta}\right) \mathrm{d} s+\sum_{k=2}^{n} \int_{(k-1) \delta}^{k \delta}\left(\frac{\mathrm{E} X_{s}^{+}}{s}-\frac{\mathrm{E} X_{k \delta}^{+}}{k \delta}\right) \mathrm{d} s .
\end{aligned}
$$


We respectively denote by $u(\delta)$ and $v(\delta)$ the first and second terms on the right-hand side of the last equality. We easily deduce from Proposition 2 that, if $\sigma>0, u(\delta)=O(\sqrt{\delta})$ and, if $\sigma=0$ and $\int_{|x| \leq 1}|x| v(\mathrm{~d} x)<\infty, u(\delta)=O(\delta)$. We also have

$$
\frac{u(\delta)}{\sqrt{\delta}}=\int_{0}^{\delta} \frac{\mathrm{E} X_{s}^{+}}{s \sqrt{\delta}} \mathrm{d} s-\frac{\mathrm{E} X_{\delta}^{+}}{\sqrt{\delta}}=\int_{0}^{1} \frac{1}{\sqrt{s}} \frac{\mathrm{E} X_{s \delta}^{+}}{\sqrt{s \delta}} \mathrm{d} s-\frac{\mathrm{E} X_{\delta}^{+}}{\sqrt{\delta}},
$$

and we easily deduce from Lemma 4 that, if $\sigma=0, u(\delta)=o(\sqrt{\delta})$.

We now study $v(\delta)$. For $s \geq 0$, let $\tilde{X}_{s}=X_{s}-\alpha s$, where $\alpha=\mathrm{E} X_{1}$. Then, $\tilde{X}$ is a martingale and, for a fixed $s \geq 0,\left(\tilde{X}_{\tau}+\alpha s\right)_{\tau \geq 0}^{+}$is a submartingale, because $x \rightarrow x^{+}$is a convex function. So, for $s \in[(k-1) \delta, \delta]$,

$$
\mathrm{E} X_{s}^{+}=\mathrm{E}\left(\tilde{X}_{s}+\alpha s\right)^{+} \leq \mathrm{E}\left(\tilde{X}_{k \delta}+\alpha s\right)^{+}
$$

Hence,

$$
\begin{aligned}
v(\delta)= & \sum_{k=2}^{n} \int_{(k-1) \delta}^{k \delta}\left(\frac{\mathrm{E} X_{s}^{+}}{s}-\frac{\mathrm{E} X_{k \delta}^{+}}{k \delta}\right) \mathrm{d} s \\
\leq & \sum_{k=2}^{n} \int_{(k-1) \delta}^{k \delta}\left(\frac{\mathrm{E}\left(\tilde{X}_{k \delta}+\alpha s\right)^{+}}{s}-\frac{\mathrm{E}\left(\tilde{X}_{k \delta}+\alpha k \delta\right)^{+}}{k \delta}\right) \mathrm{d} s \\
= & \sum_{k=2}^{n} \int_{(k-1) \delta}^{k \delta} \mathrm{E}\left(\tilde{X}_{k \delta}+\alpha k \delta\right)^{+}\left(\frac{1}{s}-\frac{1}{k \delta}\right) \mathrm{d} s \\
& +\sum_{k=2}^{n} \int_{(k-1) \delta}^{k \delta} \frac{\mathrm{E}\left(\tilde{X}_{k \delta}+\alpha s\right)^{+}-\mathrm{E}\left(\tilde{X}_{k \delta}+\alpha k \delta\right)^{+}}{s} \mathrm{~d} s .
\end{aligned}
$$

Using the inequality $\left|x^{+}-y^{+}\right| \leq|x-y|$, we obtain

$$
\begin{aligned}
v(\delta) & \leq \sum_{k=2}^{n} \mathrm{E} X_{k \delta}^{+}\left(\log \left(\frac{k}{k-1}\right)-\frac{1}{k}\right)+\sum_{k=2}^{n} \int_{(k-1) \delta}^{k \delta} \frac{|\alpha|(k \delta-s)}{s} \mathrm{~d} s \\
& =\sum_{k=2}^{n} \mathrm{E} X_{k \delta}^{+}\left(\log \left(1+\frac{1}{k-1}\right)-\frac{1}{k}\right)+\sum_{k=2}^{n} \int_{(k-1) \delta}^{k \delta}|\alpha|\left(\frac{k \delta}{s}-1\right) \mathrm{d} s \\
& \leq \sum_{k=2}^{n} \mathrm{E} X_{k \delta}^{+}\left(\frac{1}{k-1}-\frac{1}{k}\right)+\sum_{k=2}^{n}|\alpha| \delta\left(k \log \left(\frac{k}{k-1}\right)-1\right) \\
& \leq \sum_{k=2}^{n} \mathrm{E} X_{k \delta}^{+} \frac{1}{k(k-1)}+|\alpha| \delta \sum_{k=2}^{n}\left(\frac{k}{k-1}-1\right) \\
& =\sum_{k=2}^{n} \mathrm{E} X_{k \delta}^{+} \frac{1}{k(k-1)}+|\alpha| \delta \sum_{k=2}^{n} \frac{1}{k-1} .
\end{aligned}
$$

Now, if $\sigma=0$ and $\int_{|x| \leq 1}|x| v(\mathrm{~d} x)<\infty$, we know from Proposition 2 that $\mathrm{E} X_{k \delta}^{+} \leq C k \delta$ for 
some $C>0$, so that

$$
\begin{aligned}
v(\delta) & \leq C \delta \sum_{k=2}^{n} \frac{1}{k-1}+|\alpha| \delta \sum_{k=2}^{n} \frac{1}{k-1} \\
& \leq(C \delta+|\alpha|)(1+\log (n-1)) \\
& =O\left(\frac{\log (n)}{n}\right),
\end{aligned}
$$

completing the proof of statement 3 of the theorem.

For the other cases, let $f(s)=\mathrm{E}\left(X_{s}^{+}\right) / \sqrt{s}$, so that

$$
\sum_{k=2}^{n} \mathrm{E} X_{k \delta}^{+} \frac{1}{k(k-1)}=\sqrt{\delta} \sum_{k=2}^{n} f(k \delta) \frac{1}{\sqrt{k}(k-1)} .
$$

We know from Proposition 2 that $f$ is bounded on $[0, t]$, so the first statement of Theorem 2 now follows from the convergence of the series $\sum 1 / k^{3 / 2}$.

In order to prove the second statement (i.e. the case $\sigma=0$ ), we observe that

$$
\sum_{k=2}^{n} f(k \delta) \frac{1}{\sqrt{k}(k-1)} \rightarrow 0 \quad \text { as } n \rightarrow \infty
$$

as follows easily from $\lim _{s \rightarrow 0} f(s)=0$ (cf. Lemma 4).

Remark 2. The second result of Theorem 2 is optimal in the following sense: for any $\varepsilon>0$, there exists a Lévy process $X$ satisfying $\sigma=0$, such that

$$
\lim _{n \rightarrow+\infty} n^{1 / 2+\varepsilon} \mathrm{E}\left(M_{t}-M_{t}^{n}\right)=+\infty .
$$

More precisely, if $X$ is a stable process of order $\alpha$, with $\alpha \in(1,2)$, we have

$$
\lim _{n \rightarrow \infty} n^{1 / \alpha} \mathrm{E}\left(M_{t}-M_{t}^{n}\right)=-t^{1 / \alpha} \zeta\left(1-\frac{1}{\alpha}\right) \mathrm{E} X_{1}^{+} .
$$

The proof can be found in [7].

We extend the results obtained in the previous section for the case of compound Poisson processes to the case of Lévy processes with infinite activity and finite variation.

Theorem 3. Let $X$ be an integrable Lévy process with generating triplet $(\gamma, 0, v)$. Suppose that $\int_{|x| \leq 1}|x||\log (|x|)| v(\mathrm{~d} x)<\infty$ and $v(\mathbb{R})=+\infty$. Then

$$
\mathrm{E}\left(M_{t}-M_{t}^{n}\right)=\left(\left(\gamma_{0}^{+}+\int_{\mathbb{R}} x^{+} v(\mathrm{~d} x)\right) t-\mathrm{E} X_{t}^{+}\right) \frac{1}{2 n}+o\left(\frac{1}{n}\right) .
$$

Lemma 5. If $X$ is a finite variation Lévy process with infinite activity and $\gamma_{0} \neq 0$, then

$$
\int_{0}^{t} \mathrm{~d} s \int_{0}^{1} \mathrm{~d} u \frac{1}{s}\left|\mathrm{P}\left[X_{s} \geq 0\right]-\mathrm{P}\left[X_{s u} \geq 0\right]\right|<\infty .
$$


Proof. We first consider the case $\gamma_{0}<0$. Recall that, since $X$ has finite variation, we have, with probability $1, \lim _{t \rightarrow 0} X_{t} / t=\gamma_{0}$; therefore, $\mathrm{P}\left(R_{0}>0\right)=1$, where

$$
R_{0}=\inf \left\{t>0 \mid X_{t}>0\right\}
$$

and $\int_{0}^{t} s^{-1} \mathrm{P}\left(X_{s}>0\right) \mathrm{d} s<\infty$ (see [12, Section 47], especially Theorem 47.2). Set

$$
I=\int_{0}^{t} \mathrm{~d} s \int_{0}^{1} \mathrm{~d} u \frac{1}{s}\left|\mathrm{P}\left[X_{s} \geq 0\right]-\mathrm{P}\left[X_{s u} \geq 0\right]\right|
$$

Note that, since $X$ has infinite activity, we have $\mathrm{P}\left[X_{s}=0\right]=0$ for all $s>0$ (see [12, Theorem 27.4]), so that

$$
\begin{aligned}
I & \leq \int_{0}^{t} \frac{1}{s} \mathrm{P}\left[X_{s} \geq 0\right] \mathrm{d} s+\int_{0}^{t} \mathrm{~d} s \int_{0}^{1} \mathrm{~d} u \frac{1}{s} \mathrm{P}\left[X_{s u} \geq 0\right] \\
& =\int_{0}^{t} \frac{1}{s} \mathrm{P}\left[X_{s}>0\right] \mathrm{d} s+\int_{0}^{t} \mathrm{~d} s \int_{0}^{1} \mathrm{~d} u \frac{1}{s} \mathrm{P}\left[X_{s u}>0\right] .
\end{aligned}
$$

So, we need to prove that $\int_{0}^{t} \mathrm{~d} s \int_{0}^{1} \mathrm{~d} u s^{-1} \mathrm{P}\left[X_{s u}>0\right]<\infty$. We have

$$
\begin{aligned}
\int_{0}^{t} \mathrm{~d} s \int_{0}^{1} \mathrm{~d} u \frac{1}{s} \mathrm{P}\left[X_{s u}>0\right] & =\int_{0}^{t} \mathrm{~d} s \int_{0}^{s} \mathrm{~d} u \frac{1}{s^{2}} \mathrm{P}\left[X_{u}>0\right] \\
& =\int_{0}^{t} \frac{1}{s^{2}}\left(\int_{0}^{s} \mathrm{P}\left[X_{u}>0\right] \mathrm{d} u\right) \mathrm{d} s \\
& =\left[-\frac{1}{s}\left(\int_{0}^{s} \mathrm{P}\left[X_{u}>0\right] \mathrm{d} u\right)\right]_{0}^{t}+\int_{0}^{t} \frac{1}{s} \mathrm{P}\left[X_{s}>0\right] \mathrm{d} s
\end{aligned}
$$

But, for any $s>0$,

$$
\left|\frac{1}{s}\left(\int_{0}^{s} \mathrm{P}\left[X_{u} \geq 0\right] \mathrm{d} u\right)\right| \leq 1 .
$$

So, again using $\int_{0}^{t} s^{-1} \mathrm{P}\left[X_{s}>0\right] \mathrm{d} s<\infty$, we conclude that

$$
\int_{0}^{t} \mathrm{~d} s \int_{0}^{1} \mathrm{~d} u \frac{1}{s} \mathrm{P}\left[X_{s u} \geq 0\right]<\infty .
$$

Consider now $\gamma_{0}>0$. Let $\tilde{X}$ be the dual process of $X$ (e.g. $\left.\tilde{X}=-X\right)$. Then $\gamma_{0}^{\tilde{X}}=-\gamma_{0}$, and so $\gamma_{0}^{\tilde{X}}<0$. Thus,

$$
\begin{aligned}
I & =\int_{0}^{t} \mathrm{~d} s \int_{0}^{1} \mathrm{~d} u \frac{1}{s}\left|\mathrm{P}\left[X_{s}<0\right]-\mathrm{P}\left[X_{s u}<0\right]\right| \\
& =\int_{0}^{t} \mathrm{~d} s \int_{0}^{1} \mathrm{~d} u \frac{1}{s}\left|\mathrm{P}\left[\tilde{X}_{s} \geq 0\right]-\mathrm{P}\left[\tilde{X}_{s u} \geq 0\right]\right| \\
& <\infty .
\end{aligned}
$$

Proof of Theorem 3. By Proposition 1 we have

$$
\mathrm{E}\left(M_{t}-M_{t}^{n}\right)=\int_{0}^{t} \frac{\mathrm{E} X_{s}^{+}}{s} \mathrm{~d} s-\sum_{k=1}^{n} \frac{\mathrm{E} X_{k \delta}^{+}}{k} .
$$


Define

$$
h(s)=\frac{\mathrm{E} X_{s}^{+}}{s}, \quad s \in[0, t] .
$$

In order to prove the theorem, we need to show that $h$ is absolutely continuous (cf. Lemma 2). We will first show that the derivative (in the sense of distributions) of $s \mapsto \mathrm{E} X_{s}^{+}$is given by the function

$$
\frac{\mathrm{d}}{\mathrm{d} s} \mathrm{E}\left(X_{s}\right)^{+}=\gamma_{0} \mathrm{P}\left[X_{s} \geq 0\right]+\int_{\mathbb{R}} \mathrm{E}\left(\left(X_{s}+y\right)^{+}-\left(X_{s}\right)^{+}\right) v(\mathrm{~d} y), \quad s \in(0, t) .
$$

We first consider a continuously differentiable function $f$ with bounded derivative. Since $X$ is a finite variation process, Itô's formula reduces to

$$
f\left(X_{s}\right)=f(0)+\gamma_{0} \int_{0}^{s} f^{\prime}\left(X_{\tau}\right) \mathrm{d} \tau+\sum_{0 \leq \tau \leq s}\left(f\left(X_{\tau}\right)-f\left(X_{\tau^{-}}\right)\right),
$$

so that

$$
\mathrm{E} f\left(X_{s}\right)=f(0)+\gamma_{0} \mathrm{E} \int_{0}^{s} f^{\prime}\left(X_{\tau}\right) \mathrm{d} \tau+\mathrm{E} \sum_{0 \leq \tau \leq s}\left(f\left(X_{\tau}\right)-f\left(X_{\tau^{-}}\right)\right) .
$$

It follows from the compensation formula (see the preliminaries of [3]) that if

$$
\mathrm{E}\left(\int_{0}^{s} \mathrm{~d} \tau \int_{\mathbb{R}}\left|f\left(X_{\tau}+y\right)-f\left(X_{\tau}\right)\right| v(\mathrm{~d} y)\right)<\infty
$$

then

$$
\mathrm{E} \sum_{0 \leq \tau \leq s}\left(f\left(X_{\tau}\right)-f\left(X_{\tau^{-}}\right)\right)=\mathrm{E}\left(\int_{0}^{s} \mathrm{~d} \tau \int_{\mathbb{R}}\left(f\left(X_{\tau}+y\right)-f\left(X_{\tau}\right)\right) v(\mathrm{~d} y)\right) .
$$

Since $f$ is a Lipschitz function and $X$ is integrable, condition (6) is satisfied and we have

$$
\begin{aligned}
\mathrm{E} f\left(X_{s}\right) & =f(0)+\gamma_{0} \mathrm{E} \int_{0}^{s} f^{\prime}\left(X_{\tau}\right) \mathrm{d} \tau+\mathrm{E}\left(\int_{0}^{s} \mathrm{~d} \tau \int_{\mathbb{R}}\left(f\left(X_{\tau}+y\right)-f\left(X_{\tau}\right)\right) \nu(\mathrm{d} y)\right) \\
& =f(0)+\mathrm{E}\left(\gamma_{0} \int_{0}^{s} f^{\prime}\left(X_{\tau}\right) \mathrm{d} \tau+\int_{0}^{s} \mathrm{~d} \tau \int_{\mathbb{R}}\left(f\left(X_{\tau}+y\right)-f\left(X_{\tau}\right)\right) \nu(\mathrm{d} y)\right) .
\end{aligned}
$$

Now, for $\varepsilon>0$, define

$$
f_{\varepsilon}(x)=\frac{x}{2}+\frac{\sqrt{\varepsilon+x^{2}}}{2}, \quad x \in \mathbb{R} .
$$

Note that $f_{\varepsilon}$ is continuously differentiable and

$$
f_{\varepsilon}^{\prime}(x)=\frac{1}{2}+\frac{x}{2 \sqrt{\varepsilon+x^{2}}}, \quad x \in \mathbb{R},
$$

so that $\left\|f_{\varepsilon}^{\prime}\right\|_{\infty} \leq 1$. We can write

$$
\mathrm{E} f_{\varepsilon}\left(X_{s}\right)=\frac{\sqrt{\varepsilon}}{2}+\mathrm{E}\left(\gamma_{0} \int_{0}^{s} f_{\varepsilon}^{\prime}\left(X_{\tau}\right) \mathrm{d} \tau+\int_{0}^{s} \mathrm{~d} \tau \int_{\mathbb{R}}\left(f_{\varepsilon}\left(X_{\tau}+y\right)-f_{\varepsilon}\left(X_{\tau}\right)\right) \nu(\mathrm{d} y)\right) .
$$

Note that the function $f_{\varepsilon}$ converges uniformly to $x \rightarrow x^{+}$when $\varepsilon$ goes to 0 . Also, for any $x \neq 0$,

$$
\lim _{\varepsilon \rightarrow 0} f_{\varepsilon}^{\prime}(x)=\mathbf{1}_{\{x \geq 0\}}
$$


Moreover, for any $\tau>0, \mathrm{P}\left[X_{\tau} \neq 0\right]=1$ (because $X$ has infinite activity), and, for any $x \in \mathbb{R}$,

$$
x^{+} \leq f_{\varepsilon}(x) \leq \frac{x}{2}+\frac{\sqrt{\varepsilon}+|x|}{2} \leq x^{+}+\frac{\sqrt{\varepsilon}}{2} .
$$

By dominated convergence, we obtain

$$
\begin{aligned}
\mathrm{E}\left(X_{s}\right)^{+} & =\mathrm{E}\left(\gamma_{0} \int_{0}^{s} \mathbf{1}_{\left\{X_{\tau} \geq 0\right\}} \mathrm{d} \tau+\int_{0}^{s} \mathrm{~d} \tau \int_{\mathbb{R}}\left(\left(X_{\tau}+y\right)^{+}-\left(X_{\tau}\right)\right)^{+} \nu(\mathrm{d} y)\right) \\
& =\gamma_{0} \int_{0}^{s} \mathrm{P}\left[X_{\tau} \geq 0\right] \mathrm{d} \tau+\int_{0}^{s} \mathrm{~d} \tau \int_{\mathbb{R}} \mathrm{E}\left(\left(X_{\tau}+y\right)^{+}-\left(X_{\tau}\right)^{+}\right) \nu(\mathrm{d} y) .
\end{aligned}
$$

Hence,

$$
\frac{\mathrm{d}}{\mathrm{d} s} \mathrm{E}\left(X_{s}\right)^{+}=\gamma_{0} \mathrm{P}\left[X_{s} \geq 0\right]+\int_{\mathbb{R}} \mathrm{E}\left(\left(X_{s}+y\right)^{+}-\left(X_{s}\right)^{+}\right) v(\mathrm{~d} y) .
$$

Now, we have

$$
\begin{aligned}
h(s) & -\int_{\mathbb{R}} y^{+} v(\mathrm{~d} y) \\
& =\frac{\mathrm{E}\left(X_{s}\right)^{+}}{s}-\int_{\mathbb{R}} y^{+} v(\mathrm{~d} y) \\
& =\frac{1}{s} \int_{0}^{s}\left(\gamma_{0} \mathrm{P}\left[X_{u} \geq 0\right]+\int_{\mathbb{R}} \mathrm{E}\left(\left(X_{u}+y\right)^{+}-X_{u}^{+}\right) v(\mathrm{~d} y)\right) \mathrm{d} u-\int_{\mathbb{R}} y^{+} v(\mathrm{~d} y) \\
& =\frac{\gamma_{0}}{s} \int_{0}^{s} \mathrm{P}\left[X_{u} \geq 0\right] \mathrm{d} u+\frac{1}{s} \int_{0}^{s} \int_{\mathbb{R}} \mathrm{E}\left(\left(X_{u}+y\right)^{+}-X_{u}^{+}-y^{+}\right) v(\mathrm{~d} y) \mathrm{d} u
\end{aligned}
$$

But

$$
\begin{aligned}
\left(X_{u}+y\right)^{+}-X_{u}^{+}-y^{+} & =\left(X_{u}+y\right) \mathbf{1}_{\left\{X_{u}+y>0\right\}}-X_{u} \mathbf{1}_{\left\{X_{u}>0\right\}}-y \mathbf{1}_{\{y>0\}} \\
& =X_{u}\left(\mathbf{1}_{\left\{X_{u}+y>0\right\}}-\mathbf{1}_{\left\{X_{u}>0\right\}}\right)+y\left(\mathbf{1}_{\left\{X_{u}+y>0\right\}}-\mathbf{1}_{\{y>0\}}\right) \\
& =-\left|X_{u}\right| \mathbf{1}_{\left\{y X_{u}<0,|y|>\left|X_{u}\right|\right\}}-|y| \mathbf{1}_{\left\{y X_{u}<0,|y| \leq\left|X_{u}\right|\right\}} \\
& =-\left|X_{u}\right| \wedge|y| \mathbf{1}_{\left\{y X_{u}<0\right\}} .
\end{aligned}
$$

So

$$
h(s)-\int_{\mathbb{R}} y^{+} v(\mathrm{~d} y)=\frac{\gamma_{0}}{s} \int_{0}^{s} \mathrm{P}\left[X_{u} \geq 0\right] \mathrm{d} u-\frac{1}{s} \int_{0}^{s} \int_{\mathbb{R}} \mathrm{E}\left(\left(\left|X_{u}\right| \wedge|y|\right) \mathbf{1}_{\left\{y X_{u}<0\right\}}\right) v(\mathrm{~d} y) \mathrm{d} u .
$$

It is now clear that $h$ is continuous on $(0,+\infty)$, and that its derivative is given by

$$
h^{\prime}(s)=u_{s}+v_{s}+w_{s},
$$

where

$$
\begin{aligned}
& u_{s}=\frac{\gamma_{0}}{s} \mathrm{P}\left[X_{s} \geq 0\right]-\frac{\gamma_{0}}{s^{2}} \int_{0}^{s} \mathrm{P}\left[X_{u} \geq 0\right] \mathrm{d} u, \\
& v_{s}=-\frac{1}{s} \int_{\mathbb{R}} \mathrm{E}\left(\left|X_{s}\right| \wedge|y| \mathbf{1}_{\left\{y X_{s}<0\right\}}\right) v(\mathrm{~d} y), \\
& w_{s}=\frac{1}{s^{2}} \int_{0}^{s} \int_{\mathbb{R}} \mathrm{E}\left(\left|X_{u}\right| \wedge|y| \mathbf{1}_{\left\{y X_{u}<0\right\}}\right) \nu(\mathrm{d} y) \mathrm{d} u .
\end{aligned}
$$


We will now show that

$$
\int_{0}^{t}\left|h^{\prime}(s)\right| \mathrm{d} s<\infty .
$$

We have $u_{s}=0$ if $\gamma_{0}=0$, and, for $\gamma_{0} \neq 0$, we can write

$$
\begin{aligned}
\left|u_{s}\right| & =\left|\frac{\gamma_{0}}{s} \mathrm{P}\left[X_{s} \geq 0\right]-\frac{\gamma_{0}}{s^{2}} \int_{0}^{s} \mathrm{P}\left[X_{u} \geq 0\right] \mathrm{d} u\right| \\
& \leq \frac{\left|\gamma_{0}\right|}{s} \int_{0}^{1}\left|\mathrm{P}\left[X_{s} \geq 0\right]-\mathrm{P}\left[X_{s u} \geq 0\right]\right| \mathrm{d} u .
\end{aligned}
$$

Hence, by Lemma 5,

$$
\int_{0}^{t}\left|u_{s}\right| \mathrm{d} s<\infty .
$$

Besides, using the concavity of the function $x \in \mathbb{R}^{+} \rightarrow x \wedge|y|$ and Proposition 2, we obtain

$$
\begin{aligned}
\left|v_{s}\right| & \leq \frac{1}{s} \int_{\mathbb{R}} \mathrm{E}\left(\left|X_{s}\right| \wedge|y|\right) \mathbf{1}_{\left\{y X_{s}<0\right\}} v(\mathrm{~d} y) \\
& \leq \frac{1}{s} \int_{\mathbb{R}} \mathrm{E}\left(\left|X_{s}\right| \wedge|y|\right) v(\mathrm{~d} y) \\
& \leq \frac{1}{s} \int_{\mathbb{R}}\left(\mathrm{E}\left|X_{s}\right|\right) \wedge|y| v(\mathrm{~d} y) \\
& \leq \frac{1}{s} \int_{\mathbb{R}}(c s) \wedge|y| v(\mathrm{~d} y),
\end{aligned}
$$

where the positive constant $c$ comes from Proposition 2 . Now, let $\hat{v}_{s}=(1 / s) \int_{\mathbb{R}}(c s) \wedge|y| v(\mathrm{~d} y)$. Using Fubini's theorem, we have

$$
\begin{aligned}
\int_{0}^{t}\left|\hat{v}_{s}\right| \mathrm{d} s & =\int_{\mathbb{R}} v(\mathrm{~d} y) \int_{0}^{t} \frac{\mathrm{d} s}{s}(c s) \wedge|y| \\
& \leq c \int_{\mathbb{R}} \int_{0}^{|y| / c} \mathrm{~d} s v(\mathrm{~d} y)+\int_{\mathbb{R}} \int_{|y| / c}^{t} \frac{1}{s}|y| \mathbf{1}_{\{|y| \leq c t\}} \mathrm{d} s v(\mathrm{~d} y) \\
& =\int_{\mathbb{R}}|y| v(\mathrm{~d} y)+\int_{\mathbb{R}} \log \left(\frac{c t}{|y|}\right)|y| \mathbf{1}_{\{|y| \leq c t\}} v(\mathrm{~d} y) \\
& =\int_{\mathbb{R}}|y| v(\mathrm{~d} y)+\int_{|y| \leq c t} \log \left(\frac{c t}{|y|}\right)|y| v(\mathrm{~d} y) \\
& <\infty
\end{aligned}
$$

Note that the last integral is finite, owing to the assumption on the Lévy measure. For the term $w_{s}$, we have

$$
\begin{aligned}
\left|w_{s}\right| & \leq \frac{1}{s^{2}} \int_{0}^{s} \int_{\mathbb{R}}(c u) \wedge|y| v(\mathrm{~d} y) \mathrm{d} u \\
& \leq \frac{1}{s^{2}} \int_{0}^{s} \int_{\mathbb{R}}(c s) \wedge|y| v(\mathrm{~d} y) \mathrm{d} u \\
& =\frac{1}{s} \int_{\mathbb{R}}(c s) \wedge|y| v(\mathrm{~d} y) \\
& =\hat{v}_{s} .
\end{aligned}
$$


We deduce that

$$
\int_{0}^{t}\left|w_{s}\right| \mathrm{d} s<\infty .
$$

Therefore, we have proved that $h$ is absolutely continuous. Using Lemma 2 and Proposition 1 , we complete the proof.

\section{Extension of the Asmussen-Glynn-Pitman theorem}

The continuity correction results of Broadie et al. [5] for lookback options within the BlackScholes model are based on a result due to Asmussen et al. [2] about the weak convergence of the normalized difference between the continuous and discrete maximums of Brownian motion (see [2, Theorem 1]). In this section we extend this result to Lévy processes with finite activity and a nontrivial Brownian component, i.e. a Lévy process with generating triplet $\left(\gamma, \sigma^{2}, \nu\right)$, where $\sigma^{2}>0$ and $v$ is a finite measure.

The following statement is a reformulation of the Asmussen-Glynn-Pitman theorem. It can be deduced from a careful reading of the proof of Theorem 1 of [2] (see, in particular, pages 879-883 and Remark 2).

Theorem 4. Consider four real numbers $a, b, x$, and $y$, with $0 \leq a<b$. Let $\beta=\left(\beta_{t}\right)_{a \leq t \leq b}$ be a Brownian bridge from $x$ to $y$ over the time interval $[a, b]$ (so that $\beta_{a}=x$ and $\beta_{b}=y$ ), and let $t$ be a fixed positive number. Denote by $M$ the supremum of $\beta$ and, for any positive integer $n$, by $M^{n}$ the discrete supremum associated with a mesh of size $t / n$, so that

$$
M=\sup _{a \leq t \leq b} \beta_{t} \text { and } M^{n}=\sup _{k \in I_{n}} \beta_{k t / n}
$$

where

$$
I_{n}=\left\{\begin{array}{l|l}
k \in \mathbb{N} & \frac{k t}{n} \in[a, b]
\end{array} .\right.
$$

Then, as $n$ goes to $\infty$, the pair $\left(\sqrt{n}\left(M-M^{n}\right), \beta\right)$ converges in distribution to the pair $(\sqrt{t} W, \beta)$, where $W$ is independent of $\beta$ and can be written as

$$
W=\min _{j \in \mathbb{Z}} \check{R}(U+j) .
$$

Here $(\check{R}(t))_{t \in \mathbb{R}}$ is a two-sided three-dimensional Bessel process (i.e. $\check{R}(t)=R_{1}(t)$ for $t \geq 0$ and $\check{R}(t)=R_{2}(-t)$ for $t<0$, where $R_{1}$ and $R_{2}$ are independent copies of the usual threedimensional Bessel process, starting from 0$)$, and $U$ is uniformly distributed on $[0,1]$ and independent of $\check{R}$.

We can now state and prove the main result of this section.

Theorem 5. Let $X=\left(X_{t}\right)_{t \geq 0}$ be a finite activity Lévy process with generating triplet $\left(\gamma, \sigma^{2}, v\right)$ satisfying $\sigma^{2}>0$. For a fixed positive real number $t$, consider the continuous supremum of $X$ over $[0, t]$ and, for any positive integer $n$, the discrete supremum associated with a mesh of size $t / n$, that is,

$$
M_{t}=\sup _{0 \leq s \leq t} X_{s} \text { and } M_{t}^{n}=\sup _{k=0,1, \ldots, n} X_{k t / n} .
$$

Then, as $n$ goes to $\infty$, the pair $\left(\sqrt{n}\left(M_{t}-M_{t}^{n}\right), X^{(t)}=\left(X_{s}\right)_{0 \leq s \leq t}\right)$ converges in distribution to the pair $\left(\sigma \sqrt{t} W, X^{(t)}\right)$, where $W$ is independent of $X^{(t)}$ and given by (7). 
Note that, in the above statement, $X^{(t)}$ is viewed as a random variable with values in the space of càdlàg functions defined on the interval $[0, t]$, which can be endowed with the Skorokhod topology.

Remark 3. Theorem 5 might be true in the infinite activity case, as long as the Lévy process has a Brownian part (i.e. $\sigma^{2}>0$ ). However, our method, based on conditioning with respect to the jump times, cannot be easily extended to this setting. Such an extension would probably require an analogue for Lévy processes of the representation of Brownian motion and its maximum in terms of Bessel processes.

Proof of Theorem 5. We will prove that, for any bounded and continuous function $f$ and any bounded random variable $Z$ which is measurable with respect to the $\sigma$-algebra generated by the random variables $X_{s}, 0 \leq s \leq t$, we have

$$
\lim _{n \rightarrow \infty} \mathrm{E}\left(f\left(\sqrt{n}\left(M_{t}-M_{t}^{n}\right)\right) Z\right)=\mathrm{E}(f(\sigma \sqrt{t} W)) \mathrm{E}(Z) .
$$

Since $X$ is a finite activity process, it admits the representation

$$
X_{s}=\gamma_{0} s+\sigma B_{s}+\sum_{j=1}^{N_{s}} Y_{j}, \quad s \geq 0,
$$

where $B$ is a standard Brownian motion, $N$ is a Poisson process with intensity $\lambda=v(\mathbb{R})$, and the random variables $Y_{j}$ are i.i.d. with distribution $\nu / v(\mathbb{R})$. Note that $B, N$, and the $Y_{j} \mathrm{~s}$ are independent.

By conditioning with respect to $N_{t}$ we have

$$
\mathrm{E}\left(f\left(\sqrt{n}\left(M_{t}-M_{t}^{n}\right)\right) Z\right)=\sum_{m=0}^{\infty} \mathrm{E}\left(f\left(\sqrt{n}\left(M_{t}-M_{t}^{n}\right)\right) Z \mid N_{t}=m\right) \mathrm{P}\left[N_{t}=m\right] .
$$

Note that, conditionally on $\left\{N_{t}=0, X_{t}=y\right\}$, the process $X^{(t)} / \sigma$ is a Brownian bridge from 0 to $y / \sigma$ so that, using Theorem 4 ,

$$
\lim _{n \rightarrow+\infty} \mathrm{E}\left(f\left(\sqrt{n}\left(M_{t}-M_{t}^{n}\right)\right) Z \mid N_{t}=0\right)=\mathrm{E}(f(\sigma \sqrt{t} W)) \mathrm{E}\left(Z \mid N_{t}=0\right) .
$$

For the conditional expectation given $\left\{N_{t}=m\right\}, m \geq 1$, we condition further with respect to the jump times, to the values of $X$, and to the values of the left-hand limits at the jump times. Denote by $T_{1}, T_{2}, \ldots, T_{j}, \ldots$ the jump times of the Poisson process $N$. For any numbers $0<t_{1}<t_{2}<\cdots<t_{m}<t, x_{1}, \ldots, x_{m}, y_{1}, \ldots, y_{m}, y_{m+1}$, let

$$
A_{m}=\left\{N_{t}=m, T_{i}=t_{i}, X_{T_{i}^{-}}=x_{i}, X_{T_{i}}=y_{i}, i=1, \ldots, m, X_{t}=y_{m+1}\right\} .
$$

We observe that, conditionally on $A_{m}$, the random processes $\beta^{0}, \ldots, \beta^{m}$ defined by

$$
\beta_{s}^{j}= \begin{cases}\frac{1}{\sigma} X_{s} & \text { if } s \in\left[t_{j}, t_{j+1}\right), \\ \frac{1}{\sigma} X_{t_{j+1}^{-}} & \text {if } s=t_{j+1},\end{cases}
$$


with $t_{0}=0$ and $t_{m+1}=t$, are independent Brownian bridges over the intervals $\left[t_{j}, t_{j+1}\right]$. Introduce the random variables

$$
M^{j}=\sup _{t_{j} \leq s \leq t_{j+1}} \beta_{s}^{j}, \quad M^{j, n}=\sup _{k \in I_{n}^{j}} \beta_{k t / n}^{j},
$$

where $I_{n}^{j}=\left\{k \in \mathbb{N} \mid t_{j} \leq k t / n \leq t_{j+1}\right\}$. Conditionally on $A_{m}$, the random variables $M^{j}$ are independent and each of them admits a density. Therefore, with probability 1 , one of them has to be strictly larger than the others. For $j=0, \ldots, m$, set

$$
A_{m}^{j}=\left\{M^{j}>M^{i} \text { for } i \neq j\right\} .
$$

Conditionally on $A_{m}$, we have

$$
f\left(\sqrt{n}\left(M_{t}-M_{t}^{n}\right)\right) Z=\sum_{j=0}^{m} \mathbf{1}_{A_{m}^{j}} f\left(\sqrt{n}\left(\sigma M^{j}-M_{t}^{n}\right)\right) G_{j}\left(\beta^{0}, \ldots, \beta^{m}\right)
$$

for some bounded Borel functions $G_{j}$ defined on the space $\prod_{j=0}^{m} C\left(\left[t_{j}, t_{j+1}\right]\right)$. Now, on the set $A_{m}^{j}$ we have, for large enough $n, M_{t}^{n}=\sigma M^{j, n}$. This follows from the fact that the maximum of $\beta^{j}$ is attained at an interior point of the interval $\left(t_{j}, t_{j+1}\right)$ and the fact that, for large enough $n$, some elements of $I_{n}^{j}$ are arbitrarily close to this point. Therefore, for large enough $n$, we have

$$
f\left(\sqrt{n}\left(M_{t}-M_{t}^{n}\right)\right) Z=\sum_{j=0}^{m} \mathbf{1}_{A_{m}^{j}} f\left(\sigma \varepsilon_{n}^{j}\right) G_{j}\left(\beta^{0}, \ldots, \beta^{m}\right),
$$

with $\varepsilon_{n}^{j}=\sqrt{n}\left(M^{j}-M_{t}^{j, n}\right)$. We deduce from Theorem 4 and the independence of the Brownian bridges that

$$
\begin{aligned}
\lim _{n \rightarrow \infty} \mathrm{E}\left(f\left(\sqrt{n}\left(M_{t}-M_{t}^{n}\right)\right) Z \mid A_{m}\right) & =\sum_{j=0}^{m} \lim _{n \rightarrow \infty} \mathrm{E}\left(\mathbf{1}_{A_{m}^{j}} f\left(\sigma \varepsilon_{n}^{j}\right) G_{j}\left(\beta^{0}, \ldots, \beta^{m}\right) \mid A_{m}\right) \\
& =\sum_{j=0}^{m} \mathrm{E}(f(\sigma \sqrt{t} W)) \mathrm{E}\left(\mathbf{1}_{A_{m}^{j}} G_{j}\left(\beta^{0}, \ldots, \beta^{m}\right) \mid A_{m}\right) \\
& =\mathrm{E}(f(\sigma \sqrt{t} W)) \mathrm{E}\left(Z \mid A_{m}\right) .
\end{aligned}
$$

Hence, for all $m \geq 1$,

$$
\lim _{n \rightarrow \infty} \mathrm{E}\left(f\left(\sqrt{n}\left(M_{t}-M_{t}^{n}\right)\right) Z \mid N_{t}=m\right)=\mathrm{E}(f(\sigma \sqrt{t} W)) \mathrm{E}\left(Z \mid N_{t}=m\right),
$$

so that (8) follows easily.

In order to use the convergence in distribution above, we sometimes need to switch between the limit and expected values. For that purpose, the following result of uniform integrability will be useful.

Lemma 6. Let $X$ be a finite activity Lévy process with generating triplet $\left(\gamma, \sigma^{2}, v\right)$, satisfying $\sigma>0$. Fix $t>0$, and set $\varepsilon_{n}=M_{t}-M_{t}^{n}$. Then the sequence $\left(\sqrt{n} \varepsilon_{n} \mathrm{e}^{-M_{t}}\right)_{n \geq 1}$ is uniformly integrable. If, in addition, $\mathrm{E}^{q M_{t}}<\infty$ for some $q>2$ then the sequence $\left(\sqrt{n} \varepsilon_{n} \mathrm{e}^{M_{t}}\right)_{n \geq 1}$ is uniformly integrable. 
Proof. We will prove that $\left(\sqrt{n} \varepsilon_{n} \mathrm{e}^{M_{t}}\right)_{n \geq 1}$ is uniformly integrable. The other case can be easily deduced. We will use the same notation as in the proof of Theorem 5. Note that, on the set $\left\{N_{t}=0\right\}$, we have $X_{s}=\gamma_{0} s+\sigma B_{s}$ for $0 \leq s \leq t$, so the uniform integrability of the sequence $\left(\sqrt{n} \varepsilon_{n} \mathrm{e}^{M_{t}} \mathbf{1}_{\left\{N_{t}=0\right\}}\right)_{n \geq 1}$ follows from Lemma $\overline{6}$ of [2]. On the event $\left\{N_{t} \geq 1\right\}$, we will need to rule out the case when there is no jump between two mesh points. So, we introduce the event

$\Lambda_{n}=\left\{N_{t} \geq 1\right.$ and there exists a $j \in\left\{1, \ldots, N_{t}\right\}$ such that $\left.T_{j}-T_{j-1} \leq \frac{t}{n}\right\} \cup\left\{t-T_{N_{t}} \leq \frac{t}{n}\right\}$.

Note that

$$
\mathrm{P}\left[\Lambda_{n}\right] \leq \mathrm{P}\left[t-T_{N_{t}} \leq \frac{t}{n}\right]+\mathrm{E} \sum_{j=1}^{N_{t}} \mathbf{1}_{\left\{T_{j}-T_{j-1} \leq t / n\right\}} \leq \frac{\mathrm{E} N_{t}\left(N_{t}+1\right)}{n},
$$

where we have used the inequalities $\mathrm{P}\left[t-T_{N_{t}} \leq t / n \mid N_{t}=l\right] \leq l / n$ and $\mathrm{P}\left[T_{j}-T_{j-1} \leq\right.$ $\left.t / n \mid N_{t}=l\right] \leq l / n$ (cf. [7, Proposition 5.5]). Therefore, we have, using $\varepsilon_{n} \leq M_{t}$ and Hölder's inequality,

$$
\mathrm{E}\left(\sqrt{n} \varepsilon_{n} \mathrm{e}^{M_{t}} \mathbf{1}_{\Lambda_{n}}\right) \leq \sqrt{n}\left(\mathrm{E} M_{t}^{p} \mathrm{e}^{p M_{t}}\right)^{1 / p}\left(\mathrm{P}\left(\Lambda_{n}\right)\right)^{1-1 / p}
$$

for every $p>1$. Since $\mathrm{Ee}^{q M_{t}}<\infty$ for some $q>2$, we can choose $p>2$. Hence,

$$
\lim _{n \rightarrow \infty} \mathrm{E}\left(\sqrt{n} \varepsilon_{n} \mathrm{e}^{M_{t}} \mathbf{1}_{\Lambda_{n}}\right)=0 .
$$

Now, we want to prove that the sequence $\left(\sqrt{n} \varepsilon_{n} \mathrm{e}^{M_{t}} \mathbf{1}_{\left\{N_{t} \geq 1\right\} \cap \Lambda_{n}^{\mathrm{c}}}\right)_{n \geq 1}$ is uniformly integrable.

Fix $m \geq 1$ and $t_{1}, \ldots, t_{m}$ satisfying $0<t_{1}<\cdots<t_{m}<t$. Conditionally on $\left\{N_{t}=m\right.$, $\left.T_{1}=t_{1}, \ldots, T_{m}=t_{m}\right\} \cap \Lambda_{n}^{\mathrm{c}}$, we have, with probability 1 ,

$$
\varepsilon_{n}=\sum_{j=0}^{m}\left(M^{j}-M_{t}^{n}\right) \mathbf{1}_{\left\{M^{j}>\max _{i \neq j} M^{i}\right\}},
$$

where $M^{j}=\sup _{t_{j} \leq s<t_{j+1}} X_{s}, t_{0}=0$, and $t_{m+1}=t$. Moreover, owing to the definition of $\Lambda_{n}$, each subinterval $\left[t_{j}, t_{j+1}\right)$ contains at least one mesh point. Define

$$
\begin{aligned}
& k_{j}=\min \left\{k \in\{0,1, \ldots, n\} \mid \frac{k t}{n} \geq t_{j}\right\}, \\
& l_{j}=\max \left\{k \in\{0,1, \ldots, n\} \mid \frac{k t}{n} \leq t_{j+1}\right\},
\end{aligned}
$$

and let $s^{*}$ be a point at which the supremum of $X_{s}$ over $\left[t_{j}, t_{j+1}\right)$ is attained. If $s^{*} \in\left(t_{j}, k_{j} t / n\right)$, we can write

$$
M^{j}-M_{t}^{n} \leq \sup _{s \in\left(t_{j}, k_{j} t / n\right)}\left(X_{s}-X_{k_{j} t / n}\right) .
$$

If $s^{*} \in\left(l_{j} t / n, t_{j+1}\right)$, we have

$$
M^{j}-M_{t}^{n} \leq \sup _{s \in\left(l_{j} t / n, t_{j+1}\right)}\left(X_{s}-X_{l_{j} t / n}\right)
$$

Hence,

$$
M^{j}-M_{t}^{n} \leq \delta_{n, j}+\varepsilon_{n, j}+\eta_{n, j},
$$


where

$$
\delta_{n, j}=\sup _{s \in\left(t_{j}, k_{j} t / n\right)}\left(X_{s}-X_{k_{j} t / n}\right), \quad \eta_{n, j}=\sup _{s \in\left(l_{j} t / n, t_{j+1}\right)}\left(X_{s}-X_{l_{j} t / n}\right),
$$

and

$$
\varepsilon_{n, j}=\sup _{k_{j} t / n \leq s \leq l_{j} t / n} X_{s}-\max _{k_{j} \leq k \leq l_{j}} X_{k t / n}
$$

Observe that

$$
\begin{aligned}
\delta_{n, j} & =\sup _{s \in\left(t_{j}, k_{j} t / n\right)}\left[\gamma_{0} s+\sigma B_{s}-\left(\gamma_{0} \frac{k_{j} t}{n}+\sigma B_{k_{j} t / n}\right)\right] \\
& \leq\left|\gamma_{0}\right| \frac{t}{n}+\sigma \sup _{s \in\left(t_{j}, k_{j} t / n\right)}\left|B_{s}-B_{k_{j} t / n}\right| .
\end{aligned}
$$

Similarly,

$$
\eta_{n, j} \leq\left|\gamma_{0}\right| \frac{t}{n}+\sigma \sup _{s \in\left(l_{j} t / n, t_{j+1}\right)}\left|B_{s}-B_{l_{j} t / n}\right| .
$$

Note that $\left|t_{j}-k_{j} t / n\right| \leq t / n$ and $t_{j+1}-l_{j} t / n \leq t / n$. Therefore, we easily deduce from (9) and (10) that the conditional expectations of any power of $\sqrt{n} \delta_{n, j}$ and $\sqrt{n} \eta_{n, j}$, respectively, are bounded by a constant which is independent of the conditioning. We also have

$$
\varepsilon_{n, j}=\sup _{0 \leq s \leq\left(l_{j}-k_{j}\right) t / n} \beta_{s}^{j}-\max _{0 \leq k \leq l_{j}-k_{j}} \beta_{k t / n}^{j},
$$

where $\beta_{s}^{j}=\gamma_{0} s+\sigma\left(B_{s+k_{j} t / n}-B_{k_{j} t / n}\right)$. Using Lemma 6 of [2], we see that the conditional expectation of any power of $\sqrt{n} \varepsilon_{n, j}$ is bounded by a constant which is independent of the conditioning. We conclude from this discussion that, for any $p>1$,

$$
\mathrm{E}\left(\left(\sqrt{n} \varepsilon_{n} \mathbf{1}_{\Lambda_{n}^{\mathrm{c}} \cap\left\{N_{t} \geq 1\right\}}\right)^{p} \mid N_{t}\right) \leq C_{p} N_{t}^{p},
$$

where $C_{p}$ is a deterministic constant which depends only on $p, \gamma_{0}, \sigma$, and $t$. The uniform integrability of $\sqrt{n} \varepsilon_{n} \mathrm{e}^{M_{t}}$ follows easily.

\section{Continuity correction}

In this section we extend the results of Broadie et al. [5] on lookback and hindsight options to the jump diffusion model. Let $\left(S_{t}\right)_{t \in[0, T]}$ be the price of a security modeled as a stochastic process on a filtered probability space $\left(\Omega, \mathcal{F},\left(\mathcal{F}_{t}\right)_{t \in[0, T]}, \mathrm{P}\right)$. The $\sigma$-algebra $\mathcal{F}_{t}$ represents the historical information on the price until time $t$. Under the exponential Lévy model, the process $S$ behaves as the exponential of a Lévy process

$$
S_{t}=S_{0} \mathrm{e}^{X_{t}}
$$

where $X$ is a Lévy process with generating triplet $\left(\gamma, \sigma^{2}, v\right)$. The considered probability is a risk-neutral probability, under which the process $\left(\mathrm{e}^{-(r-\delta) t} S_{t}\right)_{t \in[0, T]}$ is a martingale. The parameter $r$ is the risk-free interest rate, and $\delta$ is the dividend rate. The options we will consider in the sequel will have as underlying the asset with price $S$. We will denote by $K$ the strike price of the option (in the case of hindsight options). Table 1 gives the payoffs of lookback and hindsight options. The corresponding prices are the expected values of the discounted payoffs. 
TABLE 1: The payoffs of lookback and hindsight options.

\begin{tabular}{ccc}
\hline Option & Continuous & Discrete \\
\hline Lookback call & $S_{T}-S_{0} \mathrm{e}^{m_{T}}$ & $S_{T}-S_{0} \mathrm{e}^{m_{T}^{n}}$ \\
Lookback put & $S_{0} \mathrm{e}^{M_{T}}-S_{T}$ & $S_{0} \mathrm{e}_{T}^{M_{T}^{n}}-S_{T}$ \\
Hindsight call & $\left(S_{0} \mathrm{e}^{M_{T}}-K\right)^{+}$ & $\left(S_{0} \mathrm{e}^{M_{T}^{n}}-K\right)^{+}$ \\
Hindsight put & $\left(K-S_{0} \mathrm{e}^{m_{T}}\right)^{+}$ & $\left(K-S_{0} \mathrm{e}^{m_{T}^{n}}\right)^{+}$ \\
\hline
\end{tabular}

The random variables $m_{T}$ and $m_{T}^{n}$ in Table 1 satisfy

$$
m_{T}=\inf _{0 \leq s \leq T} X_{s}, \quad m_{T}^{n}=\min _{0 \leq k \leq n} X_{k \Delta t},
$$

where $\Delta t=T / n$. The results we are going to show depend on the assumptions made on the process $X$. That is why we need to introduce the following assumptions.

(H1) $X$ is an integrable Lévy process with finite activity, satisfying $\sigma>0$, and there exists $q>2$ such that $\mathrm{Ee}^{q M_{T}}<\infty$. Recall that the condition $\mathrm{Ee}^{q M_{T}}<\infty$ is equivalent to $\int_{x>1} \mathrm{e}^{q x} v(\mathrm{~d} x)<\infty$

(H2) $X$ is an integrable Lévy process with finite activity, satisfying $\sigma>0$.

Let $W$ be the random variable defined in Theorem 4 . We set $\beta_{1}=\mathrm{E} W=-\zeta(1 / 2) / \sqrt{2 \pi}$, where $\zeta$ is the Riemann zeta function.

At a given time $t \in[0, T)$, the value of the continuous lookback put is given by

$$
V\left(S_{+}\right)=\mathrm{e}^{-r(T-t)} E \max \left(S_{+}, \max _{t \leq u \leq T} S_{u}\right)-S_{t} \mathrm{e}^{-\delta(T-T)},
$$

where $S_{+}=\max _{0 \leq u \leq t} S_{u}$ is the predetermined maximum. The continuous value of the lookback call will depend similarly on $S_{-}=\min _{0 \leq u \leq t} S_{u}$ (the predetermined minimum) and on $\min _{t \leq u \leq T} S_{u}$. The price of the discrete lookback put at the $k$ th fixing date is given by

$$
V_{n}\left(S_{+}\right)=\mathrm{e}^{-r \Delta(n-k)} E \max \left(S_{+}, \max _{k \leq j \leq n} S_{j \Delta t}\right)-S_{k \Delta t} \mathrm{e}^{-\delta(n-k) \Delta t},
$$

where $S_{+}=\max _{0 \leq j \leq k} S_{j \Delta t}$. The discrete call value will depend similarly on $S_{-}=$ $\min _{0 \leq j \leq k} S_{j \Delta t}$ and on $\min _{k \leq j \leq n} S_{j \Delta t}$.

Proposition 3. The price of a discrete lookback option at the kth fixing date and the price of the continuous lookback option at $k \Delta t$ satisfy

$$
V_{n}\left(S_{ \pm}\right)=\mathrm{e}^{\mp \beta_{1} \sigma \sqrt{T / n}} V\left(S_{ \pm} \mathrm{e}^{ \pm \beta_{1} \sigma \sqrt{T / n}}\right) \pm\left(\mathrm{e}^{\mp \beta_{1} \sigma \sqrt{T / n}}-1\right) \mathrm{e}^{-\delta(T-t)} S_{t}+o\left(\frac{1}{\sqrt{n}}\right),
$$

and

$$
V\left(S_{ \pm}\right)=\mathrm{e}^{ \pm \beta_{1} \sigma \sqrt{T / n}} V_{n}\left(S_{ \pm} \mathrm{e}^{\mp \beta_{1} \sigma \sqrt{T / n}}\right) \pm\left(\mathrm{e}^{ \pm \beta_{1} \sigma \sqrt{T / n}}-1\right) \mathrm{e}^{-\delta(T-t)} S_{t}+o\left(\frac{1}{\sqrt{n}}\right),
$$

where the upper and lower signs in ' \pm ' and $\mp$ ' respectively apply to puts and calls. The relations for the put are true under (H1), and those for the call are true under (H2). 
These formulae are the same as those found by Broadie et al. [5] for the Black-Scholes model.

Remark 4. If the process $X$ is an integrable Lévy process with generating triplet $(\gamma, 0, v)$, satisfying $v(\mathbb{R})<\infty$, then the price of a discrete lookback option and its continuous version at time $k \Delta t$ satisfy,

1. for the call,

$$
V_{n}\left(S_{-}\right)=V\left(S_{-}\right)+\frac{\alpha}{n}+o\left(\frac{1}{n}\right),
$$

where the constant $\alpha$ can be derived explicitly,

2. for the put, if there exists $\beta>1$ such that $\mathrm{Ee}^{\beta M_{T}}<\infty$ then

$$
V_{n}\left(S_{+}\right)=V\left(S_{+}\right)+o\left(\frac{1}{n^{(\beta-1) / \beta}}\right) .
$$

The proof of these results can be found in [7].

Proof of Proposition 3. Since we have Theorem 5 and Lemma 6, the proof of the above proposition is similar to the proof of Theorem 3 of [5]. For example, to relate discrete lookback puts with respect to continuous lookback puts, we need to prove that, for $x \in \mathbb{R}$,

$$
\mathrm{E}\left(\mathrm{e}^{M_{T}^{n}}-x\right)^{+}=\mathrm{e}^{-\beta_{1} \sigma \sqrt{T / n}} \mathrm{E}\left(\mathrm{e}^{M_{T}}-\mathrm{e}^{\beta_{1} \sigma \sqrt{T / n}} x\right)^{+}+o\left(\frac{1}{\sqrt{n}}\right) .
$$

In fact, we have to show first that

$$
\begin{aligned}
\mathrm{E}\left(\mathrm{e}^{M_{T}}-x\right)^{+}= & \mathrm{E}\left(\mathrm{e}^{M_{T}}-\mathrm{e}^{M_{T}^{n}}\right) \mathbf{1}_{\left\{\mathrm{e}^{\left.M_{T}>x\right\}}\right.}+\mathrm{E}\left(\mathrm{e}^{M_{T}^{n}}-x\right)^{+} \\
& +\mathrm{E}\left(\mathrm{e}^{M_{T}^{n}}-x\right) \mathbf{1}_{\left\{\mathrm{e}^{M_{T}^{n}} \leq x<\mathrm{e}^{M_{T}}\right\}} .
\end{aligned}
$$

So

$$
\begin{aligned}
\mathrm{E}\left(\mathrm{e}^{M_{T}^{n}}-x\right)^{+}= & \mathrm{E}\left(\mathrm{e}^{M_{T}}-x\right)^{+}-\mathrm{E}\left(\mathrm{e}^{M_{T}}-\mathrm{e}^{M_{T}^{n}}\right) \mathbf{1}_{\left\{\mathrm{e}^{M_{T}}>x\right\}} \\
& -\mathrm{E}\left(\mathrm{e}^{M_{T}^{n}}-x\right) \mathbf{1}_{\left\{\mathrm{e}^{M_{T}^{n}} \leq x<\mathrm{e}^{M_{T}}\right\}} .
\end{aligned}
$$

But

$$
\begin{aligned}
\mathrm{E}\left|\mathrm{e}^{M_{T}^{n}}-x\right| \mathbf{1}_{\left\{\mathrm{e}^{M_{T}^{n}} \leq x<\mathrm{e}^{\left.M_{T}\right\}}\right.} & \leq \mathrm{E}\left(\mathrm{e}^{M_{T}}-\mathrm{e}^{M_{T}^{n}}\right) \mathbf{1}_{\left\{\mathrm{e}^{M_{T}^{n}} \leq x<\mathrm{e}^{M_{T}}\right\}} \\
& \leq \mathrm{E}\left(M_{T}-M_{T}^{n}\right) \mathrm{e}^{M_{T}} \mathbf{1}_{\left\{\mathrm{e}^{M_{T}^{n}} \leq x<\mathrm{e}^{M_{T}}\right\}} .
\end{aligned}
$$

Moreover, the sequence

$$
\left(\sqrt{n}\left(M_{T}-M_{T}^{n}\right) \mathrm{e}^{M_{T}} \mathbf{1}_{\left\{\mathrm{e}^{M_{T}^{n}} \leq x<\mathrm{e}^{\left.M_{T}\right\}}\right.}\right)_{n \geq 1}
$$

is uniformly integrable (by Lemma 6). So

$$
\lim _{n \rightarrow+\infty} \mathrm{E} \sqrt{n}\left(M_{T}-M_{T}^{n}\right) \mathrm{e}^{M_{T}} \mathbf{1}_{\left\{\mathrm{e}^{M_{T}^{n}} \leq x<\mathrm{e}^{\left.M_{T}\right\}}\right.}=0 .
$$

On the other hand, using Theorem $5,\left(\sqrt{n}\left(\mathrm{e}^{M_{T}}-\mathrm{e}^{M_{T}^{n}}\right) f\left(M_{T}\right)\right)_{n \geq 0}$ converges weakly to $\sigma \sqrt{T} W \mathrm{e}^{M_{T}} f\left(M_{T}\right)$ for any measurable function $f$. So, using Lemma 6 again, we have

$$
\mathrm{E}\left(\mathrm{e}^{M_{T}}-\mathrm{e}^{M_{T}^{n}}\right) \mathbf{1}_{\left\{\mathrm{e}^{\left.M_{T}>x\right\}}\right.}=\sigma \beta_{1} \sqrt{\frac{T}{n}} \mathrm{E}^{M_{T}} \mathbf{1}_{\left\{\mathrm{e}^{\left.M_{T}>x\right\}}\right.}+o\left(\frac{1}{\sqrt{n}}\right) .
$$


Thus,

$$
\begin{aligned}
\mathrm{E}\left(\mathrm{e}^{M_{T}^{n}}-x\right)^{+}= & \mathrm{E}\left(\mathrm{e}^{M_{T}}-x\right)^{+}-\sigma \beta_{1} \sqrt{\frac{T}{n}} \mathrm{E}^{M_{T}} \mathbf{1}_{\left\{\mathrm{e}^{\left.M_{T}>x\right\}}\right.}+o\left(\frac{1}{\sqrt{n}}\right) \\
= & \mathrm{e}^{-\sigma \beta_{1} \sqrt{T / n}} \mathrm{E}\left(\mathrm{e}^{M_{T}}-x \mathrm{e}^{\sigma \beta_{1} \sqrt{T / n}}\right) \mathbf{1}_{\left\{\mathrm{e}^{\left.M_{T}>x\right\}}\right.}+o\left(\frac{1}{\sqrt{n}}\right) \\
= & \mathrm{e}^{-\sigma \beta_{1} \sqrt{T / n}} \mathrm{E}\left(\mathrm{e}^{M_{T}}-x \mathrm{e}^{\sigma \beta_{1} \sqrt{T / n}}\right) \mathbf{1}_{\left\{x<\mathrm{e}^{M_{T}} \leq x \mathrm{e}^{\left.\sigma \beta_{1} \sqrt{T / n}\right\}}\right.} \\
& +\mathrm{e}^{-\sigma \beta_{1} \sqrt{T / n}} \mathrm{E}\left(\mathrm{e}^{M_{T}}-x \mathrm{e}^{\sigma \beta_{1} \sqrt{T / n}}\right)^{+}+o\left(\frac{1}{\sqrt{n}}\right) .
\end{aligned}
$$

But, we can show that

$$
\mathrm{E}\left(\mathrm{e}^{M_{T}}-x \mathrm{e}^{\sigma \beta_{1} \sqrt{T / n}}\right) \mathbf{1}_{\left\{x<\mathrm{e}^{M_{T}} \leq x \mathrm{e}^{\sigma \beta_{1} \sqrt{T / n}}\right\}}=o\left(\frac{1}{\sqrt{n}}\right) .
$$

Hence,

$$
\mathrm{E}\left(\mathrm{e}^{M_{T}^{n}}-x\right)^{+}=\mathrm{e}^{-\sigma \beta_{1} \sqrt{T / n}} \mathrm{E}\left(\mathrm{e}^{M_{T}}-x \mathrm{e}^{\sigma \beta_{1} \sqrt{T / n}}\right)^{+}+o\left(\frac{1}{\sqrt{n}}\right) .
$$

The other cases can be derived in the same way. Detailed proofs are given in [7].

For hindsight options, we have similar results as for the lookback case. The price of a continuous hindsight call option at time $t$ with a predetermined maximum $S_{+}$and strike $K$ is

$$
V\left(S_{+}, K\right)=\mathrm{e}^{-r(T-t)} \mathrm{E}\left(\max \left(S_{+}, \max _{t \leq u \leq T} S_{u}\right)-K\right)^{+} .
$$

Similarly, for the put, we have

$$
V\left(S_{-}, K\right)=\mathrm{e}^{-r(T-t)} \mathrm{E}\left(K-\min \left(S_{-}, \min _{t \leq u \leq T} S_{u}\right)\right)^{+} .
$$

The discrete versions at the $k$ th fixing date are

$$
V_{n}\left(S_{+}, K\right)=\mathrm{e}^{-r \Delta t(n-k)} \mathrm{E}\left(\max \left(S_{+}, \max _{k \leq j \leq n} S_{j \Delta t}\right)-K\right)^{+}
$$

and

$$
V_{n}\left(S_{-}, K\right)=\mathrm{e}^{-r \Delta t(n-k)} \mathrm{E}\left(K-\min \left(S_{-}, \min _{k \leq j \leq n} S_{j \Delta t}\right)\right)^{+} .
$$

Proposition 4. The prices of a discrete hindsight option at the kth fixing date and its continuous version at $k \Delta t$ satisfy

$$
V_{n}\left(S_{ \pm}, K\right)=\mathrm{e}^{\mp \beta_{1} \sigma \sqrt{T / n}} V\left(S_{ \pm} \mathrm{e}^{ \pm \beta_{1} \sigma \sqrt{T / n}}, K \mathrm{e}^{ \pm \beta_{1} \sigma \sqrt{T / n}}\right)+o\left(\frac{1}{\sqrt{n}}\right)
$$

and

$$
V\left(S_{ \pm}, K\right)=\mathrm{e}^{ \pm \beta_{1} \sigma \sqrt{T / n}} V_{n}\left(S_{ \pm} \mathrm{e}^{\mp \beta_{1} \sigma \sqrt{T / n}}, K \mathrm{e}^{\mp \beta_{1} \sigma \sqrt{T / n}}\right)+o\left(\frac{1}{\sqrt{n}}\right),
$$

where the upper and lower signs in ' \pm ' and $\mp$ ' respectively apply to calls and puts. The relations for the call are true under (H1), and those for the put are true under (H2).

To explain the above proposition, we can say that, in order to price a continuous or discrete hindsight option respectively using a discrete or continuous hindsight option, we must shift the predetermined extremum and the strike. Proposition 4 can be deduced from Proposition 3 , thanks to the relations between lookback and hindsight options. 


\section{Upper bounds}

In the infinite activity case and if there is no Brownian part, the prices of the discrete and continuous calls are close to each other. The following proposition is a consequence of Theorem 2 and Theorem 3.

Proposition 5. Suppose that $X$ is an integrable infinite activity Lévy process with generating triplet $(\gamma, 0, v)$. Then the prices of a discrete call option at the kth fixing date and its continuous version at $k \Delta t$ satisfy the following assertions.

1. We have

$$
V_{n}\left(S_{-}\right)=V\left(S_{-}\right)+o\left(\frac{1}{\sqrt{n}}\right)
$$

2. If $\int_{|x| \leq 1}|x| v(\mathrm{~d} x)<\infty$,

$$
V_{n}\left(S_{-}\right)=V\left(S_{-}\right)+O\left(\frac{\log (n)}{n}\right) .
$$

3. If $\int_{|x| \leq 1}|x| \log (|x|) v(\mathrm{~d} x)<\infty$,

$$
V_{n}\left(S_{-}\right)=V\left(S_{-}\right)+O\left(\frac{1}{n}\right) .
$$

In the put case, the error between continuous and discrete prices depends on the integrability of the exponential of the supremum of the Lévy process driving the underlying asset.

Theorem 6. Suppose that $X$ is an infinite activity Lévy process with generating triplet $(\gamma, 0, v)$ and that there exists $\beta>1$ such that $\mathrm{E}^{\beta M_{T}}<\infty$. Then the price of a discrete put option at the kth fixing date and its continuous version at $k \Delta t$ satisfy the following assertions.

1. For any $\varepsilon>0$, we have

$$
V_{n}\left(S_{+}\right)=V\left(S_{+}\right)+O\left(\frac{1}{n^{(\beta-1) / 2 \beta-\varepsilon}}\right) .
$$

2. If $\int_{|x| \leq 1}|x| v(\mathrm{~d} x)<\infty$, for any $\varepsilon>0$, we have

$$
V_{n}\left(S_{+}\right)=V\left(S_{+}\right)+O\left(\left(\frac{\log (n)}{n}\right)^{(\beta-1) / \beta-\varepsilon}\right) .
$$

3. If $\int_{|x| \leq 1}|x| \log (|x|) \nu(\mathrm{d} x)<\infty$, for any $\varepsilon>0$, we have

$$
V_{n}\left(S_{+}\right)=V\left(S_{+}\right)+O\left(\frac{1}{n^{(\beta-1) / \beta-\varepsilon}}\right) .
$$

The main technical difficulty with the proof of Theorem 6 consists of deducing an estimate of $\mathrm{E}\left(\mathrm{e}^{M_{T}}-\mathrm{e}^{M_{T}^{n}}\right)$ from an estimate of $\mathrm{E}\left(M_{T}-M_{T}^{n}\right)$. In fact, the theorem can be deduced from the following lemma.

Lemma 7. Assume that $X$ is an infinite activity Lévy process with generating triplet $(\gamma, 0, v)$ and that there exists $\beta>1$ such that $\mathrm{E}^{\beta M_{T}}<\infty$. Then, for any $\varepsilon>0$, there exists a constant $C_{\varepsilon}$ such that

$$
\mathrm{E}\left(\mathrm{e}^{M_{T}}-\mathrm{e}^{M_{T}^{n}}\right) \leq C_{\varepsilon}\left(\mathrm{E}\left(M_{T}-M_{T}^{n}\right)\right)^{(\beta-1) / \beta-\varepsilon} .
$$


Proof. By the convexity of the exponential function we have

$$
\mathrm{e}^{M_{T}}-\mathrm{e}^{M_{T}^{n}} \leq\left(M_{T}-M_{T}^{n}\right) \mathrm{e}^{M_{T}} .
$$

So, by Hölder's inequality,

$$
\mathrm{E}\left(\mathrm{e}^{M_{T}}-\mathrm{e}^{M_{T}^{n}}\right) \leq\left(\mathrm{Ee}^{\beta M_{T}}\right)^{1 / \beta}\left(\mathrm{E}\left(M_{T}-M_{T}^{n}\right)^{\beta /(\beta-1)}\right)^{(\beta-1) / \beta} .
$$

Note that $\mathrm{E}^{\beta M_{T}}<\infty$ implies that $\mathrm{E} M_{T}^{q}<\infty$ for any $q>0$. Let $\rho \in(0,1)$. Then we have

$$
\begin{aligned}
\mathrm{E}\left(M_{T}-M_{T}^{n}\right)^{\beta /(\beta-1)} & =\mathrm{E}\left(M_{T}-M_{T}^{n}\right)^{\rho}\left(M_{T}-M_{T}^{n}\right)^{\beta /(\beta-1)-\rho} \\
& =\mathrm{E}\left(M_{T}-M_{T}^{n}\right)^{\rho}\left(M_{T}-M_{T}^{n}\right)^{(\beta(1-\rho)+\rho) /(\beta-1)} \\
& \leq\left(\mathrm{E}\left(M_{T}-M_{T}^{n}\right)\right)^{\rho}\left(\mathrm{E}\left(M_{T}-M_{T}^{n}\right)^{(\beta(1-\rho)+\rho) /(\beta-1)(1-\rho)}\right)^{1-\rho} .
\end{aligned}
$$

Hence, from the fact that

$$
\lim _{n \rightarrow+\infty} \mathrm{E}\left(M_{T}-M_{T}^{n}\right)^{(\beta(1-\rho)+\rho) /(\beta-1)(1-\rho)}=0,
$$

there exists a constant $C>0$ such that

$$
\begin{aligned}
\mathrm{E}\left(\mathrm{e}^{M_{T}}-\mathrm{e}^{M_{T}^{n}}\right) & \leq C\left(\mathrm{E}\left(M_{T}-M_{T}^{n}\right)\right)^{\rho(\beta-1) / \beta} \\
& =C\left(\mathrm{E}\left(M_{T}-M_{T}^{n}\right)\right)^{(\beta-1) / \beta-(1-\rho)(\beta-1) / \beta} .
\end{aligned}
$$

Then, for any $\varepsilon>0$, there exists a constant $C_{\varepsilon}>0$ such that

$$
\mathrm{E}\left(\mathrm{e}^{M_{T}}-\mathrm{e}^{M_{T}^{n}}\right) \leq C_{\varepsilon}\left(\mathrm{E}\left(M_{T}-M_{T}^{n}\right)\right)^{(\beta-1) / \beta-\varepsilon} .
$$

When the Lévy process driving the underlying asset has no positive jumps, we obtain tighter estimates.

Proposition 6. Let $X$ be a Lévy process with generating triplet $\left(\gamma, \sigma^{2}, v\right)$. We assume that $X$ has no positive jump $(v(0,+\infty)=0)$, that $\int_{-1 \leq x<0}|x| v(\mathrm{~d} x)<\infty$, and that there exists $\beta>1$ such that $\mathrm{E}^{\beta M_{T}}<\infty$. Then, the price of a discrete put lookback at the kth fixing date and its continuous version at time $k \Delta t$ satisfy the following assertions.

1. If $\sigma=0$,

$$
V_{n}\left(S_{+}\right)=V\left(S_{+}\right)+O\left(\frac{1}{n}\right) .
$$

2. If $\sigma>0$,

$$
V_{n}\left(S_{+}\right)=V\left(S_{+}\right)+O\left(\frac{\log (n)}{\sqrt{n}}\right) .
$$

Proposition 6 is based on the estimation of the moments of $M_{T}-M_{T}^{n}$, which can be performed when there are no positive jumps.

Lemma 8. Let $X$ be a Lévy process with generating triplet $\left(\gamma, \sigma^{2}, v\right)$, satisfying

$$
\int_{|x| \leq 1}|x| v(\mathrm{~d} x)<\infty
$$


We suppose that $X$ has no positive jumps. Then, for any $\beta>1$,

1. if $\sigma=0$,

$$
\mathrm{E}\left(M_{T}-M_{T}^{n}\right)^{\beta}=O\left(\frac{1}{n^{\beta}}\right)
$$

2. if $\sigma>0$,

$$
\mathrm{E}\left(M_{T}-M_{T}^{n}\right)^{\beta}=O\left(\left(\frac{\log (n)}{\sqrt{n}}\right)^{\beta}\right)
$$

Proof. We have

$$
\begin{aligned}
M_{T}-M_{T}^{n} & =\sup _{0 \leq s \leq T} X_{s}-\max _{0 \leq k \leq n} X_{k T / n} \\
& =\max _{1 \leq k \leq n} \sup _{(k-1) T / n \leq s \leq k T / n} X_{s}-\max _{0 \leq k \leq n} X_{k T / n} \\
& \leq \max _{1 \leq k \leq n} \sup _{(k-1) T / n \leq s \leq k T / n} X_{S}-\max _{1 \leq k \leq n} X_{(k-1) T / n} \\
& \leq \max _{1 \leq k \leq n}\left(\sup _{(k-1) T / n \leq s \leq k T / n} X_{S}-X_{(k-1) T / n}\right),
\end{aligned}
$$

where the random variables $\left(\sup _{(k-1) T / n \leq s \leq k T / n} X_{s}-X_{(k-1) T / n}\right)_{1 \leq k \leq n}$ are i.i.d., with the same distribution as $\sup _{0 \leq s \leq T / n} X_{s}$. But, since $X$ has no positive jumps, we have (see (4))

$$
\sup _{0 \leq s \leq T / n} X_{s} \leq \sup _{0 \leq s \leq T / n}\left(\gamma_{0} s+\sigma B_{s}\right) \leq \frac{\left|\gamma_{0}\right| T}{n}+\sigma \sup _{0 \leq s \leq T / n} B_{s} .
$$

We can easily deduce the first result of the lemma $(\sigma=0)$. In the case $\sigma>0$, we have

$$
\begin{aligned}
\sup _{0 \leq s \leq T / n} X_{s} & \leq \frac{1}{\sqrt{n}}\left(\frac{\left|\gamma_{0}\right| T}{\sqrt{n}}+\sigma \sqrt{n} \sup _{0 \leq s \leq T / n} B_{s}\right) \\
& \leq \frac{1}{\sqrt{n}}\left(\left|\gamma_{0}\right| T+\sigma \sqrt{n} \sup _{0 \leq s \leq T / n} B_{s}\right) \\
& \stackrel{\mathrm{D}}{=} \frac{1}{\sqrt{n}}\left(\left|\gamma_{0}\right| T+\sigma \sup _{0 \leq s \leq T} B_{S}\right) .
\end{aligned}
$$

Let $\left(V_{k}\right)_{1 \leq k \leq n}$ be i.i.d. random variables with the same distribution as $\left|\gamma_{0}\right| T+\sigma \sup _{0 \leq s \leq T} B_{s}$. Then we have

$$
\mathrm{E}\left(M_{T}-M_{T}^{n}\right)^{\beta} \leq\left(\frac{1}{\sqrt{n}}\right)^{\beta} \mathrm{E} \max _{1 \leq k \leq n} V_{k}^{\beta} .
$$

Let $g$ be the function defined as

$$
g(x)=(\log (x))^{\beta}, \quad x>1 .
$$

The function $g$ is concave and nondecreasing on the set $\left[\mathrm{e}^{\beta-1},+\infty\right)$. So we have

$$
\begin{aligned}
\mathrm{E} \sup _{1 \leq k \leq n} V_{k}^{\beta} & =\mathrm{E} \sup _{1 \leq k \leq n} g\left(\mathrm{e}^{V_{k}}\right) \\
& =\mathrm{E} g\left(\sup _{1 \leq k \leq n} \mathrm{e}^{V_{k}}\right) \quad \text { (because } g \text { is nondecreasing) }
\end{aligned}
$$




$$
\begin{aligned}
& \leq \mathrm{E} g\left(\sup _{1 \leq k \leq n} \mathrm{e}^{\max \left(V_{k}, \beta-1\right)}\right) \quad \text { (because } g \text { is nondecreasing) } \\
& \leq g\left(\mathrm{E} \sup _{1 \leq k \leq n} \mathrm{e}^{\max \left(V_{k}, \beta-1\right)}\right) \quad \text { (by Jensen) } \\
& \leq g\left(\mathrm{E} \sum_{k=1}^{n} \mathrm{e}^{\max \left(V_{k}, \beta-1\right)}\right) \quad \text { (because } g \text { is nondecreasing) } \\
& \leq g\left(n \mathrm{E}^{\max \left(V_{1}, \beta-1\right)}\right) .
\end{aligned}
$$

Note that we have $\mathrm{E}^{\max \left(V_{1}, \beta-1\right)}<\infty$. Hence, the second result of the lemma.

Proof of Proposition 6. To prove Proposition 6, we need to show that

$$
\mathrm{E}\left(\mathrm{e}^{M_{T}}-\mathrm{e}^{M_{T}^{n}}\right)= \begin{cases}O\left(\frac{1}{n}\right) & \text { if } \sigma=0, \\ O\left(\frac{\log (n)}{\sqrt{n}}\right) & \text { if } \sigma>0 .\end{cases}
$$

But, by the convexity of the exponential function, we have

$$
\mathrm{e}^{M_{T}}-\mathrm{e}^{M_{T}^{n}} \leq \mathrm{e}^{M_{T}}\left(M_{T}-M_{T}^{n}\right) .
$$

So, using Hölder's inequality, we obtain

$$
\mathrm{E}\left(\mathrm{e}^{M_{T}}-\mathrm{e}^{M_{T}^{n}}\right) \leq\left(\mathrm{E}^{\beta M_{T}}\right)^{1 / \beta}\left(\mathrm{E}\left(M_{T}-M_{T}^{n}\right)^{\beta /(\beta-1)}\right)^{(\beta-1) / \beta} .
$$

We conclude by Lemma 8 .

Results for hindsight options are similar to those for lookback options. This is simply due to the relations between lookback and hindsight options.

\section{References}

[1] Asmussen, S. (1987). Applied Probability and Queues, John Wiley, Chichester.

[2] Asmussen, S., Glynn, P. and Pitman, J. (1995). Discretization error in simulation of one-dimensional reflecting Brownian motion. Ann. Appl. Prob. 5, 875-896.

[3] Bertoin, J. (1996). Lévy Processes. Cambridge University Press.

[4] Broadie, M., Glasserman, P. and Kou, S. (1997). A continuity correction for discrete barrier options. Math. Finance 7, 325-349.

[5] Broadie, M., Glasserman, P. and Kou, S. G. (1999). Connecting discrete and continuous path-dependent options. Finance Stoch. 3, 55-82.

[6] Cont, R. And Tankov, P. (2004). Financial Modelling with Jump Processes. Chapman \& Hall/CRC, Boca Raton, FL.

[7] Dia, E. H. A. (2010). Exotic options under exponential Lévy model. Doctoral Thesis, Université Paris-Est. Available at http://tel.archives-ouvertes.fr/tel-00520583/.

[8] Dia, E. H. A. and Lamberton, D. (2011). Continuity correction for barrier options in jump-diffusion models. To appear in SIAM J. Financial Math.

[9] Fuh, C.-D., Luo, S.-F. AND Yen, J.-F. (2010). Pricing discrete path-dependent options under a jump-diffusion model. In Seminars in Financial Statistics, Academia Sinica, 27pp.

[10] Gobet, E. And Menozzi, S. (2010). Stopped diffusion processes: boundary corrections and overshoot. Stoch. Process. Appl. 120, 130-162.

[11] Knopp, K. (1990). Theory and Applications of Infinite Series. Dover, New York.

[12] Sato, K. I. (1999). Lévy Processes and Infinitely Divisible Distributions. Cambridge University Press. 